Research Articles: Neurobiology of Disease

\title{
Patient-derived anti-NMDAR antibody disinhibits cortical neuronal networks through dysfunction of inhibitory neuron output
}

https://doi.org/10.1523/JNEUROSCI.1689-21.2022

Cite as: J. Neurosci 2022; 10.1523/JNEUROSCI.1689-21.2022

Received: 19 August 2021

Revised: 22 December 2021

Accepted: 8 February 2022

This Early Release article has been peer-reviewed and accepted, but has not been through the composition and copyediting processes. The final version may differ slightly in style or formatting and will contain links to any extended data.

Alerts: Sign up at www.jneurosci.org/alerts to receive customized email alerts when the fully formatted version of this article is published.

Copyright @ 2022 Andrzejak et al.

This is an open-access article distributed under the terms of the Creative Commons Attribution 4.0 International license, which permits unrestricted use, distribution and reproduction in any medium provided that the original work is properly attributed. 


\section{Patient-derived anti-NMDAR antibody disinhibits cortical neuronal networks}

\section{through dysfunction of inhibitory neuron output}

Abbreviated Title: anti-NMDAR antibody disinhibits cortical networks

Ewa Andrzejak ${ }^{1}$, Eshed Rabinovitch ${ }^{2}$, Jakob Kreye ${ }^{1,3}$, Harald Prüss ${ }^{1,3}$, Christian Rosenmund ${ }^{4,5}$, Noam E.

$$
\text { Ziv }^{2} \text {, Craig C. Garner }{ }^{1,5} \text {, Frauke Ackermann }{ }^{1}
$$

${ }^{1}$ German Center for Neurodegenerative Diseases, 10117 Berlin, Germany, ${ }^{2}$ Technion Faculty of Medicine, Rappaport Institute and Network Biology Research Laboratories, Fishbach Building, Technion City, Haifa, 32000, Israel, ${ }^{3}$ Department of Neurology and Experimental Neurology, Charité - Universitätsmedizin, 10117 Berlin, Germany, ${ }^{4}$ Institute of Neurophysiology, Charité - Universitätsmedizin, 10117 Berlin, Germany, ${ }^{5}$ NeuroCure Cluster of Excellence, Charité - Universitätsmedizin, 10117 Berlin, Germany

Correspondence should be addressed to Frauke Ackermann at frauke.ackermann@dzne.de or Craig Garner at craig.garner@dzne.de

Number of pages: 50

Number of figures: 9

Number of words:

- Abstract: 212 (limit: 250)

- Introduction: 600 (limit 650)

- Discussion: 1500 (limit: 1500) 


\section{8}

Author contributions: E.A., F.A., C.C.G., C.R. and N.Z. designed research; E.A. and E.R. performed research; E.A., E.R. and N.Z. analyzed data; H.P. and J.K. provided the antibodies; E.A. wrote the first draft of the paper; E.A., F.A., C.C.G., N.Z., C.R., H.P and J.K edited the paper; E.A., C.C.G. and F.A. wrote the paper. The authors declare no competing financial interest.

Acknowledgements: This work was supported by the German Center for Neurodegenerative Diseases; the Federal Government of Germany (Deutsche Forschungsgemeinschaft): SFB958 to C.C.G, EXC-2049-390688087 for the Center of Excellence NeuroCure to F.A. and C.C.G.; PR1274/2-1, PR1274/3-1, PR1274/5-1 to H.P.; Helmholtz Association (HIL-A03 to H.P.); German Federal Ministry of Education and Research (Connect-Generate 01GM1908D to H.P.); the Israel Science Foundation (1470/18) to N.E.Z.; the State of Lower-Saxony and the Volkswagen Foundation to N.E.Z.

We thank Thorsten Trimbuch and the Viral Core Facility of the Charité - Universitätsmedizin Berlin for cloning and production of viral constructs; Marisa Brockmann for electrophysiological training; Aleksandra Ichkova for valuable comments on the manuscript; Anny Kretschmer, Christine Bruns, Bettina Brokowski and Katja Poetschke for technical assistance; and the Advanced Medical Bioimaging Core Facility (AMBIO) of the Charité - Universitätsmedizin for support in acquisition of the imaging data. 
65

66 7 8

Anti-NMDAR encephalitis is a severe neuropsychiatric disorder associated with autoantibodies against NMDA receptors, which cause a variety of symptoms from prominent psychiatric and cognitive manifestations to seizures and autonomic instability. Previous studies mainly focused on hippocampal effects of these autoantibodies, helping to explain mechanistic causes for cognitive impairment. However, antibodies' effects on higher cortical network function, where they could contribute to psychosis and/or seizures, have not until now been explored in detail. Here, we employed a patient-derived monoclonal antibody targeting the NR1 subunit of NMDAR and tested its effects on in vitro cultures of rodent cortical neurons, using imaging and electrophysiological techniques. We report that this hNR1 antibody drives cortical networks to a hyper-excitable state and disrupts mechanisms stabilizing network activity such as Npas4 signaling. Network hyperactivity is in part a result of a reduced synaptic output of inhibitory neurons, as indicated by a decreased inhibitory drive and levels of pre-synaptic inhibitory proteins, specifically in inhibitory-to-excitatory neuron synapses. Importantly, on a single-cell level hNR1 antibody selectively impairs NMDARmediated currents and synaptic transmission of cortical inhibitory neurons, yet has no effect on excitatory neurons, which contrasts with its effects on hippocampal neurons. Together, these findings provide a novel, cortex-specific mechanism of antibody-induced neuronal hyper-excitability, highlighting regional specificity underlying the pathology of autoimmune encephalitis.

Key words: autoimmune encephalitis; NMDAR; autoantibodies; network excitability; cortical interneurons 
70 It is increasingly appreciated that the inadvertent activation of the immune system within CNS can

71 underlie pathogenesis of neuropsychiatric disorders. Although the exact mechanisms remain elusive,

72 autoantibodies derived from patients with autoimmune encephalitis pose a unique tool to study

73 pathogenesis of neuropsychiatric states. Our analysis reveals that autoantibody against the NMDA

74 receptor has a distinct mechanism of action in the cortex, where it impairs function of inhibitory

75 neurons leading to increased cortical network excitability, in contrast to previously described hippocampal synaptic mechanisms of information encoding, highlighting brain regional specificity.

77 Notably, similar mechanism of NMDAR-mediated inhibitory hypofunction leading to cortical disinhibition has been suggested to underlie pathology of schizophrenia, hence our data provide new evidence for common mechanisms underlying neuropsychiatric disorders. 


\section{INTRODUCTION}

92 Over the last decade, a growing number of central nervous system disorders have been linked to autoantibodies, which have been detected in patients' cerebrospinal fluid (CSF). Many of these bind synaptic and neuronal cell-surface proteins, including a variety of neuro-transmitter receptors (Dalmau and Graus 2018). One of the most prevalent forms of autoimmune encephalitis is associated with IgG antibodies that bind $\mathrm{N}$-methyl-D-aspartate receptors (NMDARs), a disease that is characterized by a rapid clinical progression and a broad range of symptoms (Dalmau et al. 2007).

Most patients present with prominent psychiatric manifestations, including psychosis, hallucinations and behavioral changes, which further progress to severe memory loss, seizures and autonomic instability. Often patients require prolonged treatment in intensive care units (Irani et al. 2010). Interestingly, most are responsive to immunotherapies and show marked recovery (Titulaer et al. 2013). A fundamental question is how such antibodies mechanistically trigger such a broad range of symptoms in patients.

With regard to NMDAR encephalitis, most studies have focused on how autoantibodies cause memory deficits, in particular within hippocampal circuits. A framework for such investigations is based on a long-history of research showing that NMDARs are ligand-gated ion channels responsible for synaptic integration and plasticity and underlie hippocampal learning and memory processes (Bliss and Collingridge 1993; Nicoll 2017). Studies with patients' CSF reveal that most NMDARautoantibodies recognize epitopes within the extracellular domain of the obligatory NR1 subunit of NMDARs (Gleichman et al. 2012). Antibody binding primarily triggers the reversible internalization of NMDARs and thereby a net decrease in surface receptor clusters in hippocampal neurons (Hughes et al. 2010; Planagumà et al. 2015). Loss of receptors has been shown to decrease synaptic NMDARmediated currents (Hughes et al. 2010; Kreye et al. 2016) and to compromise synaptic plasticity (Mikasova et al. 2012; Q. Zhang et al. 2012; Würdemann et al. 2016). These conditions are thought to 
contribute to memory deficits described in patients with NMDAR encephalitis and animal models (Planagumà et al. 2016; Malviya et al. 2017).

At present, how such antibodies could also trigger psychiatric symptoms and/or epileptic seizures is less clear, though actions at higher network levels, for instance within cortical circuits, seem likely. Intriguingly, seizures have been observed in 60\% of encephalitis patients (Bruijn et al. 2019), suggesting an increased excitability within these circuits (Manto et al. 2010). Yet there are few clues of how NMDARs autoantibodies could drive this cortical hyper-excitability. On the other hand, it has long been suggested that NMDARs are involved in the pathology of psychosis ("glutamatergic hypothesis of schizophrenia"), wherein NMDAR antagonists such as ketamine and phencyclidine (PCP) have been found to induce psychotic symptoms, as well as exacerbate them in schizophrenic patients (Krystal et al. 1994; Jentsch and Roth 1999). This has led to the hypothesis that NMDAR hypofunction, in particular on inhibitory interneurons (Belforte et al. 2010), could also cause excitation/inhibition imbalances and disinhibition of prefrontal cortical circuits (Homayoun and Moghaddam 2007a), similar to those observed in schizophrenic patients (Uhlhaas and Singer 2010). It is currently unclear, whether auto-NMDAR antibodies could similarly affect cortical interneurons and thus alter cortical network properties in patients with NMDAR encephalitis.

In the present study, we have evaluated whether a patient-derived antibody against the NR1 subunit of NMDARs has direct effects on cortical neurons. Our detailed imaging and electrophysiological analysis of cultured rodent cortical neurons revealed that this antibody increases network spiking and bursting, driving cortical neurons into a hyper-active state. Surprisingly, this hyperactivity seems to be a result of selective hypofunction of inhibitory interneuron output, specifically in inhibitory-toexcitatory neuron synapses, leading to a decrease in inhibitory drive and hyper-excitability of these cortical neuronal networks. 


\section{Preparation of cultured cortical neurons}

141

All procedures for experiments involving animals were approved by the animal welfare committee of Charité Medical University and the Berlin state government. To distinguish inhibitory and excitatory neurons in some experiments (Fig. 4-8), we used GAD67-GFP (Aneo)/+ (GAD67-GFP) mice, in which inhibitory neurons expressing GAD67 are fluorescently labeled (Tamamaki et al. 2003). In comparison to WT neurons, no changes in the electrophysiological properties of these GFP expressing neurons have been reported (Chang et al. 2014). To prepare primary cortical neuronal cultures for immunocytochemistry and mass culture patch clamp experiments, mouse WT (C57BL/6J) (RRID:IMSR_JAX:000664)(license: T0036/14) or GAD67-GFP animals (license: T0220/09) of either sex were prepared and grown on glass coverslips using the 'Banker Protocol' (Banker and Goslin 1988; Meberg and Miller 2003). In brief, astrocytes were prepared from mouse WT cortices on postnatal day zero to two (PO-2) and seeded on 6-well or 12-well plates at a density of 10,000 per $1 \mathrm{~cm}^{2}, 7-11$ days before the addition of neurons. Cortical neurons were prepared from cortices dissected from mice PO-2 brains in cold HBSS (Millipore), followed by a 45 min incubation in enzyme solution containing DMEM (Invitrogen, Thermo Fisher Scientific), cysteine (3.3 mM), $\mathrm{CaCl}_{2}(2 \mathrm{mM}), \mathrm{EDTA}$ $(1 \mathrm{mM})$, and papain $\left(20 \mathrm{U} / \mathrm{ml}\right.$, Worthington) at $37^{\circ} \mathrm{C}$. Next, the papain reaction was inhibited by incubating cortical tissue in a DMEM solution containing 10\% FCS (Thermo Fisher Scientific), BSA (38 $\mathrm{mM}$, Sigma-Aldrich), and trypsin inhibitor (95 mM) (Sigma-Aldrich) for $5 \mathrm{~min}$. Afterwards, cells were triturated in Neurobasal-A medium (2\% B27, $1 \%$ Glutamax, 0.2\% P/S; Thermo Fisher Scientific) by gentle pipetting up and down. Isolated neuronal cells were plated onto nitric acid washed and poly-Llysine-coated glass coverslips with paraffin dots, at a density of 50,000-200,000 per 12-well coverslip. After $1.5 \mathrm{~h}$, the coverslips were placed upside down onto a bed of astrocytes and co-cultured in Neurobasal-A medium at $37^{\circ} \mathrm{C}, 5 \% \mathrm{CO} 2$, for $14-18$ DIV before starting experiments. 
To create autaptic cultures for single-cell electrophysiological recordings, cortical or hippocampal neurons were plated on micro-islands of astrocyte feeder layers, generated 1 week before the neuronal culture preparations, as described previously (Arancillo et al. 2013). Neurons were plated at a lower density (4,000 cells per 6 well coverslip) to obtain a single neuron on an astrocytic island.

For calcium imaging experiments, primary mouse cortical neurons were plated on top of a 1-week old astrocyte feeder layer, on round dishes containing a cell location grid at the bottom ( $\mu$-Dish 35 $\mathrm{mm}$, high Grid-500, Ibidi), at the density of 250,000 cells per dish. The cells were cultured in medium containing minimal essential medium (MEM; Sigma-Aldrich), insulin $(25 \mathrm{mg} / \mathrm{L}$, SigmaAldrich), glucose (20 mM, Sigma-Aldrich), L-glutamine (2 mM, Sigma-Aldrich), Pen/Strep (0.2\%, Sigma-Aldrich), and NuSerum (10\%, Becton Dickinson Labware). Seven days after plating, one-half of the culture medium was replaced with feeding medium as above, but lacking NuSerum and containing $0.5 \mathrm{mM}$ of L-glutamine and 2\% B-27 supplement (Invitrogen). Approximately one-half of the medium was then replaced with the feeding medium one or two times per week.

For multi-electrode array experiments, primary cultures of rat cortical neurons were prepared as described previously (Minerbi et al. 2009), using a protocol approved by the Technion committee for the supervision of animal experiments (IL-116-08-71). Briefly, cortices of newborn P1 Wistar rats (either sex; Charles River Laboratories) were dissected, dissociated by trypsin treatment followed by trituration using a siliconized Pasteur pipette. A total of $1-1.5 \times 10^{6}$ cells were then plated on thinglass multielectrode array (MEA) dishes (Multi Channel Systems MCS), pre-coated with polyethylenimine (Sigma-Aldrich) for better cell adhesion. Subsequently, neurons were cultured at $37^{\circ} \mathrm{C}$ and $5 \% \mathrm{CO}_{2}$, and grown in medium containing minimal essential medium (MEM; Sigma-Aldrich), insulin (25 mg/L, Sigma-Aldrich), glucose (20 mM, Sigma-Aldrich), L-glutamine (2 mm, Sigma-Aldrich), gentamycin sulfate (5 mg/ml, Sigma-Aldrich), and 10\% NuSerum (Becton Dickinson Labware). At 7 DIV, one-half of the culture medium was replaced with feeding medium lacking NuSerum and 
containing $0.5 \mathrm{mM}$ of L-glutamine and 2\% B-27 supplement (Invitrogen) and then the cultures were fed in this way 3 - 4 times per week.

\section{Lentivirus production}

All lentiviral particles were provided by the Viral Core Facility of the Charite-Universitätsmedizin Berlin (https://vcf. charite.de/en/) and were prepared as described previously (Lois et al. 2002). Briefly, HEK293T (RRID:CVCL_0063) cells were cotransfected with $10 \mu \mathrm{g}$ of shuttle vector, $5 \mu \mathrm{g}$ of helper plasmid pCMVdR8.9, and $5 \mu \mathrm{g}$ of pVSV.G with X-tremeGENE 9 DNA transfection reagent (Roche Diagnostics). Virus-containing cell culture supernatant was collected after $72 \mathrm{~h}$ and filtered for purification. Aliquots were flash-frozen in liquid nitrogen and stored at $-80^{\circ} \mathrm{C}$. Neurons were infected with lentivirus at 2-4 DIV.

\section{Production of monoclonal antibodies}

The human monoclonal antibody \#003-102 is reactive to the NR1 subunit of NMDA receptors and was isolated from cerebrospinal fluid of a patient with acute NMDAR encephalitis (Kreye et al. 2016). Isotype-matched control antibody mGO53 was isolated from blood of a healthy donor (Wardemann et al. 2003). For the recombinant expression of these antibodies, the paired expression vectors encoding for the antibodies' heavy and light chain were transiently transfected in HEK293T cells and purified from cell culture supernatants, as previously described (Kreye et al. 2016). The antibody concentration was determined using an anti-human IgG ELISA following the manufacturer's instructions (3850-1AD-6, Mabtech).

\section{Immunocytochemistry of cultured neurons}

Npas4 experiment. To verify the effects of the hNR1 antibody on somatic Npas4 expression, cortical neurons 14-17 DIV were incubated with $1 \mu \mathrm{g} / \mathrm{ml}$ of hNR1 antibody for $2 \mathrm{~h}, 4 \mathrm{~h}$ or $24 \mathrm{~h}$. Alternatively, to induce expression of Npas4, neurons were treated with NMDA ( $2 \mu \mathrm{M}$, Tocris) for $2 \mathrm{~h}$. Untreated cells were used as a control. To study the effect of hNR1 and NMDAR antagonist (2R)-amino-5- 
phosphonovaleric acid (AP5) on NMDA-induced Npas4 expression, NMDA treatment was preceded by $6 \mathrm{~h}$ or $24 \mathrm{~h}$ of hNR1 $(1 \mu \mathrm{g} / \mathrm{ml})$ or $3 \mathrm{~h}$ of AP5 (100 $\mu \mathrm{M}$, Tocris) treatment. After treatment, cells were fixed with 4\% PFA in PBS for 10 min and washed 3x 5 min with PBS. Subsequently, cells were permeabilized and blocked with a solution containing $5 \%$ normal goat serum, $2 \%$ BSA and $0.1 \%$ Triton in PBS, for $1 \mathrm{~h}$. Neurons were then incubated with primary antibodies against Npas4 (1:1500, rabbit, Activity Signaling) and MAP2 (1:2000, chicken, Millipore, AB5543) in antibody solution containing $2 \%$ BSA in PBS for $2 \mathrm{~h}$ at RT. Cells were then washed $3 \times 5$ min with PBS and incubated with the differently labeled secondary antibodies (1:1000 in antibody solution, Invitrogen, Thermo Fisher Scientific), for $2 \mathrm{~h}$ at RT and washed $3 \times 5$ min with PBS. Finally, coverslips were dipped in $\mathrm{H}_{2} \mathrm{O}$ and mounted in Pro-Long Diamon Antifade Mountant (Thermo Fisher Scientific).

Synaptic/extrasynaptic $h N R 1$ labeling. Cortical neurons of GAD67-GFP animals were subjected to live staining with hNR1 and anti-human secondary antibody. Briefly, neurons were incubated with $1 \mu \mathrm{g} / \mathrm{ml}$ of $\mathrm{hNR} 1$ in original culture NBA medium (see above) for $20 \mathrm{~min}$ at $10^{\circ} \mathrm{C}$. Cells were then washed once with same medium and incubated with Alexa-568 conjugated anti-human secondary antibody (1:1000, Thermo Fisher Scientific) for $20 \mathrm{~min}$ at $10^{\circ} \mathrm{C}$. Afterwards, neurons were again washed once with culture medium, fixed with 4\% PFA, blocked, stained with antibodies against excitatory presynaptic vesicle protein VGLUT1 (1:4000, SySy 135 304, Synaptic Systems) or the inhibitory postsynaptic scaffold protein gephyrin (1:500, SySy 147 011, Synaptic Systems) and labeled with secondary antibodies and mounted as described earlier (see Npas4 experiment).

Inhibitory pre-synaptic proteins labeling. Cortical neurons from GAD67-GFP animals were incubated with $1 \mu \mathrm{g} / \mathrm{ml}$ of hNR1 antibody in original culture NBA medium for $0 \mathrm{~h}, 6 \mathrm{~h}$ or $24 \mathrm{~h}$ at $37^{\circ} \mathrm{C}$. Subsequently, cells were fixed with 4\% PFA, blocked, stained with antibodies against MAP2 (1:2000, AB5543, Millipore), GAD65 (1:750, MAB5406, Millipore), or VGAT (1:4000, SySy 131 003, Synaptic Systems) and labeled with secondary antibodies before mounting as described above.

\section{Image acquisition and quantification}


Immunocytochemical staining for Npas4 experiments was acquired on a Nikon Spinning Disk Confocal CSU-X microscope equipped with an air 20x Plan Apo objective lens (NA = 0.8), controlled via NIS-Elements software (Nikon). Images were then processed using ImageJ (RRID: SCR_003070). ROIs were manually drawn around cell somas. After background subtraction, the mean intensity values of the ROls were measured and normalized to the untreated control.

hNR1 labeling and staining for inhibitory pre-synaptic markers were analyzed using a spinning disc confocal microscope (Carl Zeiss Axio Oberserver.Z1 with Andor spinning disc and cobalt, omricron, ibeam laser) (Carl Zeiss, Andor) using a 63x (1.4 NA) or 100x (1.4 NA) Plan-Apochromat oil objectives and an iXon ultra (Andor) camera controlled by iQ software (RRID: SCR_014461) (Andor). Only dendrites in proximity to the soma were imaged, and inhibitory and excitatory dendrites were distinguished based on GFP signal. Images were processed using ImageJ (RRID: SCR_003070) and OpenView software (written by Prof. dr. Noam Ziv, Technion Institute, Haifa, Israel). In brief, for synaptic protein levels at GAD65 and VGAT puncta along dendrites were detected with set parameters: $6 \times 6$ pixels boxes were placed over puncta and the mean fluorescence intensities were measured, followed by subtraction of background values. For hNR1 labeling within excitatory synapses, hNR1-positive puncta were detected in a similar manner and then VGLUT1 intensities within hNR1-positive puncta were measured. A value above a threshold of $5 x$ background intensity was considered VGLUT1-positive and thus synaptic. The remaining hNR1 puncta were considered extra-synaptic. In a similar manner, boxes around detected gephyrin puncta served to measure hNR1 intensity for localization of hNR1 puncta to inhibitory synapses. Z-plane images were analyzed using Volume Viewer plugin in ImageJ. Values of all puncta per region of interest (individual dendrites) were averaged and considered one data point.

\section{Multi-electrode arrays}

Neuronal network activity was recorded continuously from MEA electrodes (59 electrodes, $30 \mu \mathrm{m}$ diameter, arranged in an $8 \times 8$ array), as described previously (L. Hazan and Ziv 2020). Briefly, cells 
grown on MEA dishes were continuously perfused with fresh feeding medium at a rate of $4 \mathrm{ml} / \mathrm{day}$ using an ultra-low-flow peristaltic pump (Instech Laboratories), and maintained at $37^{\circ} \mathrm{C}$ and $5 \% \mathrm{CO}_{2}$. After setting up the perfusion system, network activity was allowed to equilibrate for $1 \mathrm{~h}$ and then $15 \mathrm{~h}$ of baseline activity was recorded. Next, $1 \mu \mathrm{g} / \mathrm{ml}$ of hNR1 antibody, control antibody or $1 \mu \mathrm{M}$ AP5 was manually pipetted into the MEA dish and added to the perfusion system. Cell spiking was recorded in the presence of the antibodies or $1 \mu \mathrm{M}$ AP5 for $24 \mathrm{~h}$. Finally, saturating concentrations of AP5 $(50 \mu \mathrm{M})$ were added to the dish to block remaining NMDARs, and the activity of the culture was recorded for additional $6 \mathrm{~h}$. Recordings from MEA dishes were performed using a commercial 60channel headstage (inverted MEA-1060-BC, MCS) with a gain of $53 \times$ and frequency limits of $0.02-$ $8500 \mathrm{~Hz}$. This signal was further filtered with frequency limits of $150-3000 \mathrm{~Hz}$ and amplified (20x) using a filter/amplifier (FA60S-BC, MCS). Data acquisition was performed using custom software [Closed Loop Experiment Manager (CLEM)] (H. Hazan and Ziv 2017). Data were collected at $16 \mathrm{k}$ Samples/s. Action potentials were identified as negative threshold-crossing events, with the threshold calculated as $5 \times$ root-mean-square of traces recorded at the beginning of each experiment. Data were imported, converted and analyzed for spiking and bursting activity using custom scripts in MATLAB (MathWorks). Data from each MEA dish was normalized to the activity of last 3 hours of baseline recordings. To test if there is a significant increase in spiking/bursting rate due to $24 \mathrm{~h}$ treatment, for each network the average rate at the end of baseline (1h) was compared to end of $24 \mathrm{~h}$ treatment $(1 \mathrm{~h})$ by paired t-test. To quantify percentage increase in spiking/bursting rates at the beginning/end of respective treatments (24h treatment with antibodies or $1 \mu \mathrm{M} \mathrm{AP5,50 \mu M} \mathrm{AP5} \mathrm{or}$ recovery at the end of experiment), the spike/burst rate of 10 min intervals was measured.

\section{Patch clamp electrophysiology}

Whole-cell patch-clamp recordings were performed on mass or autaptic, cortical or hippocampal neurons at $14-18$ DIV, after $24 \mathrm{~h}$ incubation with $1 \mu \mathrm{g} / \mathrm{ml}$ of hNR1 or control antibody (mGo53) (Wardemann et al. 2003). Cortical neurons from GAD67-eGFP mouse line were used to distinguish 
inhibitory (GFP-positive) and excitatory (GFP-negative) cells. All recordings were obtained at $25^{\circ} \mathrm{C}$ from neurons clamped at $-70 \mathrm{mV}$ with a Multiclamp 700B amplifier (Molecular Devices) under the control of Clampex 10.4 software (Molecular Devices). Data were sampled at $10 \mathrm{kHz}$ and low-pass Bessel filtered at $3 \mathrm{kHz}$ and series resistance was typically under $15 \mathrm{M} \Omega$ and compensated at $70 \%$. During all recordings, except for chemically induced NMDA and evoked synaptic NMDA responses, neurons were immersed in standard extracellular solution consisting of $140 \mathrm{mM} \mathrm{NaCl}, 2.4 \mathrm{mM} \mathrm{KCl}$, $10 \mathrm{mM}$ HEPES, $10 \mathrm{mM}$ glucose, $2 \mathrm{mM} \mathrm{CaCl}_{2}$, and $4 \mathrm{mM} \mathrm{MgCl}_{2}$. Chemically induced whole-cell NMDA responses were measured in extracellular solution containing $0 \mathrm{mM} \mathrm{Mg}^{2+}, 0.2 \mathrm{mM} \mathrm{CaCl}_{2}$, and $10 \mu \mathrm{M}$ glycine, whereas synaptically evoked NMDAR currents were measured in extracellular solution containing $0 \mathrm{mM} \mathrm{Mg}^{2+}, 2 \mathrm{mM} \mathrm{CaCl}_{2}$, and $10 \mu \mathrm{M}$ glycine. The borosilicate glass pipettes (3-8 $\mathrm{M} \Omega$ ) were pulled with a micropipette puller device (Sutter Instruments) and filled with the internal solution containing $136 \mathrm{mM} \mathrm{KCl}, 17.8 \mathrm{mM}$ HEPES, $1 \mathrm{mM}$ EGTA, $0.6 \mathrm{mM} \mathrm{MgCl}$, 4mM ATP-Mg, $0.3 \mathrm{mM}$ GTP-Na, $12 \mathrm{mM}$ phosphocreatine, and $50 \mathrm{U} / \mathrm{ml}$ phosphocreatine kinase. All solutions were adjusted to $\mathrm{pH} 7.4$ and osmolarity of $\sim 300$ mOsm.

To selectively induce NMDA, GABA and kainate currents, NMDA (10 $\mu \mathrm{M}$, Tocris), GABA (5 $\mu \mathrm{M}$, Tocris), or kainic acid ( $20 \mu \mathrm{M}$, Tocris), were acutely applied to the neurons for 1 second by use of a fast-flow system. For synaptic responses in autaptic cultures, excitatory postsynaptic currents (EPSCs) were evoked by brief somatic depolarization of neurons from $-70 \mathrm{mV}$ to $0 \mathrm{mV}$ for $2 \mathrm{~ms}$. Synaptic NMDAR currents were measured in the presence of AMPAR antagonist NBQX (10 $\mu \mathrm{M}$, Tocris).

All mass culture recordings were performed in the presence of TTX ( $0.5 \mu \mathrm{M}$, Tocris) to block propagation of action potentials. To identify spontaneous mEPSCs and mIPSCs, neurons were additionally immersed in AP5 (50 $\mu \mathrm{M})$ and bicuculline $(20 \mu \mathrm{M}$, Tocris), or AP5 (50 $\mu \mathrm{M})$ and NBQX (10 $\mu \mathrm{M})$, respectively. Traces were recorded at a holding potential of -70 mV and were filtered at $1 \mathrm{kHz}$, mEPSCs and mIPSCs were detected by a template algorithm in Axograph $\mathrm{X}$ (Axograph Scientific). False-positive events were excluded by subtracting events detected from traces in the presence of 
311 NBQX $(10 \mu \mathrm{M})$ or bicuculline $(20 \mu \mathrm{M})$ for mEPSCs and mIPSCs, respectively. To identify excitatory neurons for detection of mIPSCs in mass cultures (Fig. 4B-F), WT mouse neurons were infected with pLenti_CamKIla_mKate2, a lentivirus expressing mKate2 under a CamKII promoter, at 3-4 DIV. For experiments detecting mEPSCs (Fig. 2E-F), GAD67-GFP line neurons were used.

Electrophysiological data were analyzed offline using Axograph X (RRID:SCR_014284) (Axograph Scientific), Excel (Microsoft), and Prism (GraphPad).

\section{Calcium imaging}

Cortical cultures grown on grid-bottomed dishes were infected with f(syn)-NES-jRCamP1b-WPRE-w, a lentivirus expressing jRCamP1b under the Synapsin promoter (Viral Core Facility, Charité Universitätsmedizin), at 4 DIV. Images were acquired at $37^{\circ} \mathrm{C}$ and a $\mathrm{CO}_{2}$ controlled environment, using Nikon Spinning Disk Confocal CSU-X microscope with an air 20x Plan Apo objective lens (NA $=0.8)$, controlled via the NIS-Elements software (Nikon). Time-lapse images were collected at $5 \mathrm{~Hz}$ using an iXon3 DU-888 Ultra camera (Andor) and $561 \mathrm{~nm}$ excitation laser. In each dish, 3 fields of view with approximately 10-20 cells were selected, and 2 min of spontaneous activity was measured twice, with 5 min interval between the runs. To study bicuculline-induced changes is network activity, bicuculline $(30 \mu \mathrm{M})$ was manually pipetted into the cell culture dish immediately after the end of the second baseline activity recording. After a 30 second waiting period, two 2-minutes imaging sessions were recorded, separated by a 5 min waiting interval.

Time-lapse image analysis and automatic time-series event detection were accomplished with a custom-written script in OpenView software (Prof. dr. Noam Ziv, Technion, Israel). In brief, ROIs were manually selected by placing boxes of $27 \times 27$ pixels over visually identified neuronal cell somas. Only active cells were included in analysis. Time series fluorescence values were converted into $\Delta \mathrm{F} / \mathrm{F}$ by calculating the ratio between the change in fluorescence signal intensity (delta F) and baseline 
fluorescence (F0). The custom-written algorithm identified the timestamps of calcium transient onset, which were then averaged per min to obtain the frequency of events.

\section{Experimental design and statistical analysis}

GraphPad Prism was used to analyze and represent data. Schematic figures were created with BioRender.com. Statistical design, sample sizes, and tests for each experiment can be found in the figure legends. All figures represent data from at least 3 independent experiments (independent cultures). Unpaired and paired t-tests and ANOVA Tukey's multiple-comparison tests were used to evaluate statistical significance.

\section{RESULTS}

\section{hNR1 antibody increases spiking and bursting of cortical networks}

Previous studies have shown that human anti-NMDAR antibodies affect NMDAR function in hippocampal neurons, helping to explain potential mechanistic causes for cognitive impairment in patients with auto-immune NMDAR encephalitis (Hughes et al. 2010; Mikasova et al. 2012; Würdemann et al. 2016). However, other symptoms centered in cortical circuits, such as psychiatric manifestations, catatonia, coma and epilepsy, have yet to be explored. We were thus interested in addressing the question of whether these antibodies can also affect cortical network function and if so, what is their functional impact? To explore these fundamental issues, we took advantage of a patient-derived monoclonal antibody \#003-102 targeting the NR1 subunit of NMDAR (hNR1) (Kreye et al. 2016) and tested its effect on primary cortical neuron cultures. We began with examining effects of hNR1 antibody on neuronal network activity by growing cortical neurons on multielectrode array (MEA) dishes for 11-14 days. This approach allows a continuous and simultaneous monitoring of activity from dozens of neurons recorded by 59 electrodes. Initially, baseline spiking activity of the neuronal network was recorded for $15 \mathrm{~h}$, afterwards $1 \mu \mathrm{g} / \mathrm{ml}$ of hNR1 or control 
antibody (ctrl) (mGO53) (Wardemann et al. 2003) were added and the spiking activity traced for another 24h (Fig. 1A, I). Remarkably, we observed a dramatic change in network spiking activity in the presence of hNR1 antibody that gradually increased over the period of $24 \mathrm{~h}$ of antibody treatment, reaching a highly active state (baseline $=1.12 \pm 0.47$, after $24 \mathrm{~h}$ hNR1 $=3.02 \pm 0.47$, paired t-test, $p=0.02$ ) (Fig. 1B, D). The effect of the control antibody was more limited, eliciting a modest, but not significant, change in network spiking activity during this period (normalized baseline $=1.01$ \pm 0.01 , after $24 \mathrm{~h} \mathrm{ctrl}=1.72 \pm 0.27,5$, paired t-test, $\mathrm{p}=0.07$ ) (Fig. 1B, C).

The observed increase in spiking caused by the hNR1 antibody is both surprising and counterintuitive, as earlier studies demonstrated that active neuronal networks commonly respond to blockage of NMDARs - by NMDAR antagonists like AP5 or ketamine - with a rapid decrease of their spiking activity (Teppola et al. 2018; Teppola, Aćimović, and Linne 2019; Emnett et al. 2013), which slowly recovers over hours, due to various slow adaptive processes (Kaufman, Reinartz, and Ziv 2014). Conceptually, this difference could be due to the fact that the hNR1 antibody only blocks $50 \%$ of the whole-cell NMDAR currents in cultured hippocampal neurons (Kreye et al. 2016; Hughes et al. 2010), while saturating concentrations of AP5 and ketamine block all active NMDARs. We therefore hypothesized that the hNR1 antibody-induced increase in spiking activity could be an adaptive response of the network to an incomplete block of NMDARs. To test this hypothesis, we carried out similar experiments by adding $1 \mu \mathrm{M}$ of the NMDA antagonist AP5 ( $1 \mu \mathrm{M}$ being the IC50 for NMDARs) instead of antibody, to block $50 \%$ of the surface NMDA receptors. Here, the presence of $1 \mu \mathrm{M}$ AP5 was associated with a modest, yet non-significant, increase in spiking activity over $24 \mathrm{~h}$ (baseline $=1.02 \pm 0.01$, after $24 \mathrm{~h} 1 \mu \mathrm{M}$ AP5 $=1.43 \pm 0.13$, paired t-test, $\mathrm{p}=0.09$ ) (Fig. 1B, E, yellow data points). As the magnitude of this change was just a fraction of that elicited by hNR1 antibody ( $43 \%$ vs $\sim 300 \%$, respectively) (Fig. 1D, F), it is possible that the underlying mechanism of the latter could be different, e.g. by affecting a subpopulation of receptors or cell types. Of note, AP5 is expected to act more quickly than the hNR1 antibody, yet as both conditions involved chronic multi- 
hour treatments, the observed outcomes are anticipated to be due to more slow adapting processes by both manipulations.

An interesting consequential question is whether networks treated with hNR1 antibody or AP5 are still responsive to pharmacological manipulations of NMDARs. This was examined by adding saturating concentrations of AP5 $(50 \mu \mathrm{M})$ at the end of the $24 \mathrm{~h}$ treatment with $\mathrm{hNR} 1$, control antibody or $1 \mu \mathrm{M}$ AP5 (Fig. 1A). Interestingly, networks treated with the control antibody or $1 \mu \mathrm{M}$ AP5 remained responsive to high concentration of AP5, as their spiking rapidly decreased (95\% and $87 \%$ reduction, respectively) (Fig. 1B, 1G). Furthermore, spiking only partially recovered to about $60 \%$ of their initial value $6 \mathrm{~h}$ later ( $\mathrm{ctrl}=61 \%, \mathrm{AP} 5=45 \%$ ) (Fig. 1F). In contrast, the spiking activity of cortical cultures treated with hNR1 antibody only partially dropped due to AP5 treatment (42\% reduction, Fig. 1G) and quickly recovered to $93 \%$ of the activity levels preceding AP5 treatment (Fig. 1H). These remained nearly fourfold higher than those observed in networks exposed to the control antibody or $1 \mu \mathrm{M}$ AP5. Together these data indicate that the addition of hNR1 antibody to cortical cultures renders the network largely insensitive to NMDAR blockage with AP5.

Subsequently, we asked whether other parameters crucial for behavior of neuronal network, such as bursting, number of spikes per burst and network synchrony, are also affected by hNR1 antibody treatment. While synchrony and number of spikes per burst were not different between the groups (data not shown), we observed a dramatic increase in network bursting activity due to hNR1 antibody treatment (baseline $=1.04 \pm 0.03$; after $24 \mathrm{~h} h N R 1=3.22 \pm 0.63$, paired t-test, $\mathrm{p}=0.027$ ) (Fig. 1J, L). Burst rate of both control antibody (baseline $=1 \pm 0.03$; after $24 \mathrm{~h}$ ctrl $=1.05 \pm 0.12$, paired t-test, $\mathrm{p}=0.72$ ) and $1 \mu \mathrm{M}$ AP5 (baseline $=1 \pm 0.04$; after $24 \mathrm{~h} 1 \mu \mathrm{M} \mathrm{AP5}=1.36 \pm 0.2$, paired ttest, $p=0.2$ ) treated networks was not changed (Fig. $1 \mathrm{~J}-\mathrm{N}$ ). Intriguingly, the hNR1 antibody-induced increase in burst rate seemed to have a more rapid onset than the increase in neuronal spiking (Fig. $1 \mathrm{~B}, \mathrm{~J})$, reaching a plateau after $\sim 7 \mathrm{~h}$ of hNR1 antibody treatment (Fig. 1J). This phenomenon of elevated burst rates is a characteristic of networks in which inhibition has been removed (Chen et al. 
2012). Interestingly, high concentrations of AP5 had a smaller effect on bursting activity than on spiking. Moreover, its immediate effects (Fig. 10), or recovery of burst rate 6h later (Fig. 1P), were not different between the treatments.

To summarize, these experiments reveal two unexpected effects of hNR1 antibody on cortical neurons: 1) substantial elevations in cortical network spiking and bursting activity and 2) a partial loss of responsiveness to NMDAR blockage by AP5, a situation that could conceivably both adversely affect network function and drive epileptic brain activity in patients.

\section{hNR1 antibody impairs NMDA-mediated Npas4 expression in cortical cultures}

Neuronal networks are complex systems whose function depends on the proper function of various neuronal cell types and regulatory mechanisms, allowing them to maintain their activity within a narrow range. Normally under increasing activity conditions, calcium influx through NMDARs signals to the cell nucleus and triggers an adaptive change in neuronal gene expression profiles, resulting in a homeostatic restoration of activity levels (Flavell and Greenberg 2008; Hardingham and Bading 2003). One such activity-dependent transcription factor, whose expression is partially triggered by calcium influx through NMDARs, is Neuronal PAS domain protein 4 (Npas4) (Bloodgood et al. 2013; Spiegel et al. 2014). It was thus of interest to know whether this transcriptional program still operates following the addition of hNR1 antibody. Specifically, we asked whether the hNR1 antibody-induced increase in network activity (Fig. 1) was associated with a corresponding increase in Npas4 expression (Fig. 2). Surprisingly, treating cortical cultures with hNR1 antibody for 2h, $4 \mathrm{~h}$ or $24 \mathrm{~h}$ did not induce the expression of Npas4, as measured by changes in fluorescence intensity of somatic Npas4 in cortical cultured neurons (Fig. 2A-D). These data indicate that a Npas4 mediated homeostatic mechanism is not active and/or cannot be engaged in the presence of the hNR1 antibody. As more direct test of the latter, we asked whether hNR1 antibody disrupts NMDA- 
mediated regulation of Npas4 expression. As expected under control conditions, the addition of NMDA to cortical cultures induced significant Npas4 expression (UT $=1 \pm 0.03$, NMDA $=2.21 \pm 0.17, p<$ 0.0001) (Fig. 2E, first and second panel, Fig 2F). However, the pre-treatment with hNR1 antibody for $6 \mathrm{~h}$ or $24 \mathrm{~h}$ blocked the increase in somatic Npas4 expression, similarly to networks pretreated with AP5 $(6 \mathrm{~h} \mathrm{NR} 1+\mathrm{NMDA}=1.28 \pm 0.04,24 \mathrm{~h} h N R 1+\mathrm{NMDA}=1.13 \pm 0.03,3 \mathrm{~h}$ AP5 + NMDA $=1.47 \pm 0.07, \mathrm{p}<$ 0.0001) (Fig. 2E-F). These data suggest that hNR1 antibody interferes with the capacity of cortical networks to decrease their activity through NMDA/Npas4 regulated gene expression, an observation that could help explain why the hNR1 antibody-induced hyperactivity is not down-regulated to homeostatic levels over time. However, less clear is whether the dysregulation of NMDA/Npas4 regulated gene expression can explain why hNR1 antibody treatment increases network activity in the first place, or whether other mechanisms are at play that more directly regulate excitatory/inhibitory balance in these networks.

\section{hNR1 antibody disinhibits cortical networks by specifically impairing output of inhibitory neurons}

Proper function of cortical networks is determined by a fine balance between excitation and inhibition. Previous studies have shown that hypofunction of NMDARs can reduce inhibition and disinhibit cortical networks (Y. Zhang, Behrens, and Lisman 2008b; Homayoun and Moghaddam 2007a). It thus seemed feasible that the hNR1 antibody-induced hyperactivity is a result of network disinhibition. To test this hypothesis, we designed a set of calcium imaging and electrophysiological experiments. For calcium imaging, neurons were initially infected at 4 DIV with a lentivirus expressing RCaMP (Dana et al. 2016), a genetically encoded calcium indicator, and allowed to grow for 10 more days (14DIV) before antibody treatment. Cortical networks of neurons were then subjected to live imaging $24 \mathrm{~h}$ after treatment with hNR1 or control antibodies. Subsequently, activity levels were measured by detecting somatic calcium transients of individual cells and compared to untreated cultures. Similar to MEA recordings (Fig. 1), in cortical cultures treated with 
the hNR1 antibody the frequency of somatic calcium events dramatically increased, as compared to both untreated networks and those treated with control antibody (UT $=10.89 \pm 0.31, \mathrm{ctrl}=12.55 \pm$ $0.43, \mathrm{hNR} 1=14.99 \pm 0.37, \mathrm{p}<0.0001)$ (Fig. 3A-C upper panels, D). Interestingly, in this experimental setup the control antibody (ctrl) also increased the frequency of calcium events compared to untreated cultures $(p=0.007)$, though to a lesser extent than the hNR1 antibody (ctrl: $15 \%, h N R 1$ : $38 \%$ increase) (Fig. 3D). These latter data suggest that this antibody is not without its effects, though the mechanism and its antigen remains unknown.

Given the high spiking and bursting frequency of cortical cultures treated with hNR1 antibody, it was of interest to explore whether such cultures could be further disinhibited, perhaps by disengaging the GABAergic system. This was accomplished by adding bicuculline $(30 \mu \mathrm{M})$, a $G A B A_{A} R$ antagonist, at the end of $24 \mathrm{~h}$ treatment with antibodies and re-imaging activity of the same neurons for a within-cell comparison (Fig. 3A-F). As expected, in untreated and control antibody treated conditions, bicuculline increased the frequency of calcium events (paired t-test, $p<0.0001, p=$ 0.035, respectively) (Fig. 3E, 3F). Strikingly, bicuculline failed to further increase the frequency of calcium events in cultures treated with the hNR1 antibody (paired t-test, $p=0.53$, Fig. 3G). These data imply that critical features of the GABAergic system and/or $\mathrm{Ca}^{2+}$-activated potassium channels, also known to be inhibited by bicuculline (Khawaled et al. 1999; Johnston 2013), could contribute to antibody-induced hyper-excitability.

As the GABAergic system is a known key regulator of excitatory and inhibitory balance, we designed several experiments to further explore possible inhibitory dysfunction in the context of hNR1 antibody-disrupted NMDAR signaling. Interestingly, studies on the 'glutamatergic hypothesis of schizophrenia' suggest that hypofunction of inhibitory drive onto excitatory pyramidal cells leads to disinhibition of cortical networks (Homayoun and Moghaddam 2007b), producing psychotic symptoms similar to those observed in patients with NMDAR encephalitis. We therefore asked whether the inhibitory drive onto excitatory neurons is also altered in cortical networks due to hNR1 
482

antibody. Here, we performed a set of patch clamp electrophysiology experiments to record miniature inhibitory post-synaptic currents (mIPSCs) from excitatory neurons in cortical cultures (Fig 4A). Excitatory neurons were identified by infecting WT neurons with pLenti_CamKII_mKate2, a lentivirus expressing mKate2 under the glutamatergic specific CamKII promoter, at DIV 2-4 (Fig. 4B). At 15-18 DIV, we recorded mIPSCs from these neurons, after $24 \mathrm{~h}$ treatment with either hNR1 or control antibody. We observed that hNR1 antibody treatment significantly decreased mIPSCS frequency $(\operatorname{ctrl}=6.42 \pm 0.37, \mathrm{hNR} 1=4.94 \pm 0.4$, unpaired $\mathrm{t}$-test, $\mathrm{p}=0.009)$ and had a tendency to decrease mIPSCs amplitude $(\mathrm{ctrl}=46.89 \pm 2.89, \mathrm{hNR} 1=40.01 \pm 2.25$, unpaired $\mathrm{t}$-test, $\mathrm{p}=0.06)$ compared to control antibodies (Fig. 4C, D) without affecting charge (Fig. 4F). These data indicate that $24 \mathrm{~h}$ hNR1 antibody treatment indeed decreased inhibitory drive onto excitatory neurons. To test whether this is caused by an overall reduction of surface GABAR function on post-synaptic excitatory neurons, we measured currents induced by bath application of GABA ( $5 \mu M)$ using a fastflow system. However, these GABA-currents were similar between control and hNR1 antibody treated cultures (Fig. 4G, H), indicating that the total pools of $G_{A B A} R$ on excitatory neurons are not altered.

As network activity is sensitive to both excitation and inhibition, we next explored whether the excitatory drive in neuronal cultures was also altered by hNR1 antibody. This was accomplished by recording miniature excitatory post-synaptic currents (mEPSCs) in the presence of antibodies from both excitatory and inhibitory cortical neurons. Interestingly, the frequency, amplitude and charge of mEPSCs onto either cell type were not affected by hNR1 antibody treatment (Fig. 4I-P). These data indicate that hNR1 antibody specifically reduces the inhibitory drive onto excitatory neurons in a cortical network, which could be causal for its overall disinhibition. 
507

508

As we observed a decrease in overall inhibition and specific changes in inhibitory neuron output, we next examined the selective effects of the hNR1 antibody on inhibitory cortical neurons in more detail. Hence, we performed patch-clamp electrophysiological recordings in an autaptic system, where single neurons are grown on an astrocytic micro-island, allowing the study of cellautonomous effects on either inhibitory or excitatory neurons (Fig. 5A). To easily distinguish between these two neuronal populations, we employed GAD67-GFP mice, in which inhibitory neurons expressing GAD67 are fluorescently labeled (Tamamaki et al. 2003). Previous groups reported no changes in electrophysiological properties of these neurons when compared to WT neurons (Chang et al. 2014). In initial experiments, we examined whether the addition of hNR1 or control antibodies $(24 \mathrm{~h})$ affect the total surface expression of the 3 main groups of ionotropic receptors responsible for synaptic transmission, by acutely bath-applying NMDA (10 $\mu M)$, GABA (5 $\mu \mathrm{M})$ or kainate $(20 \mu \mathrm{M})$ in 1 second pulses and comparing responses of inhibitory and excitatory cells. Intriguingly, we observed that in autaptic cultures of cortical neurons, hNR1 antibody treatment did not affect whole-cell NMDA currents on excitatory neurons (ctrl $=1 \pm 0.08, \mathrm{hNR} 1=$ $1.01 \pm 0.11$, unpaired t-test, $p=0.92$ ) (Fig $5 B$ ), yet it selectively decreased whole-cell NMDA currents on inhibitory neurons (ctrl $=1 \pm 0.1$, hNR1 $=0.71 \pm 0.07$, unpaired t-test, $p=0.018$ ) (Fig. $5 E)$. At the same time, total GABA- and kainate-specific currents in either cell type remained unchanged (Fig. 5G, data not shown). Next, we measured evoked synaptic responses by inducing an action potential in these cells, by the brief somatic depolarization of the neuron, and recording of the post-synaptic current that followed a few milliseconds afterwards. Intriguingly, on inhibitory neurons, synaptic inhibitory transmission (IPSCs) was significantly reduced by $\sim 41 \%$ (ctrl $=1 \pm 0.12, \mathrm{hNR} 1=0.59 \pm 0.11$, $p=0.01$ ) (Fig. 5F), whereas synaptic excitatory transmission (EPSCs) (ctrl $=1 \pm 0.13, \mathrm{hNR} 1=1.31 \pm$ 0.19 , unpaired t-test, $p=0.19$ ) (Fig. $5 \mathrm{C}$ ) and pharmacologically isolated synaptic NMDA currents (ctrl $=1 \pm 0.12, \mathrm{hNR} 1=1.38 \pm 0.23$, unpaired t-test, $\mathrm{p}=0.13$ ) (Fig. 5D) on excitatory neurons remained unchanged (Fig. 5C, D). What is more, on inhibitory neurons, the reduction of NMDA-currents and 
the amplitude of IPSCs seemed to go hand in hand, as there was a significant correlation between these two parameters among inhibitory cells (linear regression, $\mathrm{R}^{2}=0.132, \mathrm{p}=0.029$ ) (Fig. $5 \mathrm{H}$ ). These data suggest that in autaptic cortical neurons hNR1 antibody treatment primarily affects the function of NMDARs on inhibitory neurons, which in turn reduces inhibitory synaptic output by these cells. To explore the mechanism further, we examined the impact of hNR1 antibody on the spontaneous release of GABA from inhibitory neurons by measuring mIPSCs in these autaptic cultures. Here, we observed a significant decrease in mIPSCs amplitude (Fig. 5K) (ctrl $=43.87 \pm 2.81$, hNR1 $=30.72 \pm 2.46$, unpaired t-test, $p=0.0008)$ and charge (Fig. $5 \mathrm{~L})(\mathrm{ctrl}=789.7 \pm 76.58, \mathrm{hNR} 1=$ $551.9 \pm 58.94$, unpaired t-test, $p=0.016)$. Similar to neuronal network experiments (Fig. $4 G, H)$, we observed no differences in responses after bath application of GABA between autaptic neurons treated with control or hNR1 antibodies (Fig. 5G). Together, these data indicate that the hNR1 antibody specifically impairs NMDAR- and synaptic transmission of cortical inhibitory, but not excitatory neurons, in a cell-autonomous manner.

Importantly, as we observed a modest effect of the control antibody on neuronal network activity (Fig. 3), we evaluated whether it also exerts any significant effects in autaptic cultures. Here, no differences between untreated and control antibody treated cultures were observed in any of reported measures (whole-cell NMDA, GABA and kainate currents, synaptic NMDA currents and EPSCS/IPSCS) in neither excitatory nor inhibitory neurons (data not shown). These data suggest that the control antibody generally has no direct effect on synaptic function in the autaptic system.

\section{hNR1 antibody exerts different effects in cortical vs hippocampal neurons}

The lack of effect of hNR1 antibody on NMDA currents in cortical excitatory neurons was surprising, as previous studies have robustly shown that patients' CSF/IgG reduce NMDA currents of hippocampal pyramidal cells (Kreye et al. 2016; Moscato et al. 2014). To verify that the lack of effect 
on cortical neurons is not due to technical limitations of our autaptic assays, we repeated the autaptic recordings on hippocampal excitatory neurons. As reported previously (Kreye et al. 2016), treatment with hNR1 antibody selectively reduced whole-cell NMDA currents ( $36 \%$ decrease, ctrl $=1$ $\pm 0.08, \mathrm{hNR} 1=0.64 \pm 0.06$, unpaired t-test, $p=0.0008)($ Fig. $6 \mathrm{~A})$ and synaptic NMDA currents $(43 \%$ decrease, $\operatorname{ctrl}=1 \pm 0.15, \mathrm{hNR} 1=0.58 \pm 0.12$, unpaired t-test, $\mathrm{p}=0.039$ ) (Fig. 6E) in hippocampal excitatory neurons, without affecting EPSC (Fig. 6D), GABA (Fig. 6B) and kainate (Fig. 6C) currents. Importantly, these results demonstrate that hNR1 antibody shows tissue-specificity, and in cortical cultures selectively affect inhibitory, and not excitatory, neurons.

hNR1 antibody binds NMDARs within inhibitory synapses and reduce levels of inhibitory presynaptic proteins

The results from both neuronal networks (Fig. 4) and autaptic (Fig. 5) recordings suggest that hNR1 antibody causes a dysfunction of synaptic output of inhibitory neurons, represented by a reduced amplitude and/or frequency of IPSCs and mIPSCs. To explore possible presynaptic effects of the hNR1 antibody, we measured levels of proteins crucial for proper function of inhibitory pre-synapses before and after hNR1 antibody treatment by immunocytochemistry in cortical networks. Additionally, by employing GAD67-GFP mice, we distinguished between inhibitory synapses onto inhibitory (GFP-positive), and excitatory (GFP-negative) neurons (Fig. 7B, C). Two proteins are indispensable for proper neurotransmitter release within GABAergic pre-synapses: glutamate decarboxylase 65 (GAD65), which synthetizes GABA from glutamate within pre-synaptic boutons, and the vesicular GABA transporter (VGAT/VIAAT), which subsequently loads GABA into synaptic vesicles. Using quantitative immunocytochemistry, we measured the intensities of fluorescently labelled VGAT and GAD65 puncta along dendrites, to quantify their expression levels. Remarkably, a $24 \mathrm{~h}$ treatment with hNR1 antibody significantly reduced intensities of VGAT puncta (UT $=1 \pm 0.05$, $24 \mathrm{~h}$ hNR1 $=0.82 \pm 0.04$, unpaired t-test, $p=0.005$ ) (Fig. 7D, E). Moreover, this decrease was specific 
to inhibitory synapses onto excitatory neurons, and not onto inhibitory neurons (Fig. 7D, F). On the other hand, the intensities of GAD65 puncta were unchanged after $24 \mathrm{~h}$ of control antibody treatment (Fig. 7G-I). We then additionally measured GAD65 expression levels after $6 \mathrm{~h}$ of hNR1 antibody treatment. At this time-point, we observed a significant decrease in GAD65 puncta intensity (UT $=1 \pm 0.03,6 \mathrm{~h}$ hNR1 $=0.89 \pm 0.02,24 \mathrm{~h}$ hNR1 $=0.95 \pm 0.02$, ANOVA Tukey's multiplecomparison test, $\mathrm{p}=0.01$ ) (Fig. $7 \mathrm{H})$, which again was specific to inhibitory synapses onto excitatory, but not inhibitory, neurons (Fig. 7G-I). Together, these data demonstrate that the hNR1 antibody reduces levels of the pre-synaptic proteins VGAT and GAD65, specifically in inhibitory-to-excitatory neuron synapses.

Finally, we asked if the observed specific effect of hNR1 antibody on inhibitory neurons can be explained by a distinct binding pattern of the antibody in cortical cultures. One possibility could be that the antibody binds specifically to inhibitory neurons. Alternatively, the hNR1 antibody could target different sub-cellular pools of NMDARs, or even bind directly to inhibitory synapses to affect their function locally. We explored these options by analyzing the acute binding patterns of hNR1 antibody in our cortical cultures. Firstly, live neurons were incubated with hNR1 antibody for 20 minutes at $10^{\circ} \mathrm{C}$, washed, and incubated with anti-human secondary antibody for 20 minutes at $10^{\circ} \mathrm{C}$. Cells were then fixed and co-stained for the excitatory presynaptic marker VGLUT1 to verify if hNR1 antibody binds to excitatory synapses, as expected. The degree of co-localization of the hNR1 antibody puncta with VGLUT1signal was used as a measure of excitatory synaptic vs extra-synaptic antibody binding. Although the hNR1 antibody decorated all neurons present in the culture in a punctate pattern, there was a striking difference between the binding patterns on excitatory and inhibitory neurons. While on inhibitory neurons most of hNR1 antibody puncta (70\%) co-localized with VGLUT1, on excitatory neurons only half of the hNR1 antibody puncta co-localized with VGLUT1 (excit. $=47.28 \% \pm 4.53$, inhibit. $=69.75 \% \pm 4.19$, unpaired t-test, $p=0.006)($ Fig. $8 \mathrm{~A}, \mathrm{~B})$, suggesting that on excitatory neurons a larger pool of hNR1 antibody binds to structures outside of VGLUT1positive synapses. Next, we asked if hNR1 antibody can also bind NMDARs associated with inhibitory 
synapses. Here, neurons were co-stained with the hNR1 antibody and the inhibitory post-synaptic marker gephyrin to assess the degree of co-localization. Using confocal microscopy and z-stack analysis, we observed that hNR1 antibody indeed binds to a sub-population of inhibitory synapses in our cortical cultures (Fig. 8C, D). Remarkably, up to $30 \%$ of inhibitory synapses on excitatory neurons bound the hNR1 antibody, whereas this phenomenon was much less common for inhibitory synapses on inhibitory neurons (excit. $=30.84 \% \pm 2.05$, inhibit. $=11.17 \% \pm 1.52$, unpaired $\mathrm{t}$-test, $\mathrm{p}<0.0001$ ) (Fig. 9E). This preference raises the possibility that the observed functional impact of the hNR1 antibody on inhibitory synapses may be due to the direct effects of this antibody on these synapses.

\section{DISCUSSION}

Our study demonstrates that a patient-derived antibody targeting the NR1 subunit of NMDARs adversely affects the function of cortical neuronal networks, driving them into a hyper-active state (Fig. 1, 3). This increased excitability seems to be a result of reduced inhibitory neuron output onto excitatory neurons, as indicated by reduced amplitudes of IPSCs, lowered frequency of network mIPSCs and decreased levels of inhibitory pre-synaptic proteins (Fig. 9). This contrasts with the effects of this antibody on hippocampal neurons, where it primarily affects NMDAR function on excitatory neurons (Fig 6, Kreye et al., 2016). Moreover, in cortical networks hNR1 antibody interferes with NMDAR-Npas4-mediated mechanisms of stabilizing network excitability (Fig. 2). These observations provide insights into how such autoantibodies can differentially affect hippocampal versus cortical circuits, giving rise to impaired memory function or psychiatric symptoms in patients with NMDAR encephalitis, respectively. 


\section{hNR1 antibody increases cortical network activity}

Our primary observation is that hNR1 antibody causes a hyper-excitable state of cortical networks (Fig. 1, 3). Although counterintuitive, since NMDAR hypofunction should dampen glutamatergic transmission, our data are in line with clinical and molecular studies showing that seizures occur in $\sim 60 \%$ of patients with NMDAR encephalitis (Bruijn et al. 2019) and that seizure susceptibility is increased in mice exposed to patient CSF (Wright et al. 2015).

Intriguingly, in contrast to our findings (Fig. 1), two groups used MEAs to assess effects of patient CSF on cortical (Jantzen et al. 2013) or hippocampal (Koch et al. 2019) neuronal networks and reported a relative decrease in activity. Such differences could arise from varied approaches used. Both studies examined the acute effects of antibodies (10-15 mins), using patient CSF containing a mixture of antibodies. In contrast, our $46 \mathrm{~h}$ recording revealed that the presence of the hNR1 antibody leads to a gradually increasing activity, however over $24 \mathrm{~h}$ and not $15 \mathrm{~min}$. Mechanistically, this occurs in a timeframe corresponding to the rate of antibody-induced receptor internalization both described previously (Moscato et al. 2014) and observed for hNR1 (data not shown). Thus, the increase in cortical network activity by the hNR1 antibody seems to be a relevant causal mechanism for both hyper-excitability and epilepsy in patients.

A fundamental question raised by our MEA analysis is how the hNR1 antibody leads to a steady increase in network spiking? One possibility is an adaptive response of the network to a partial block of NMDARs. Blocking half of surface NMDARs (1 $\mu \mathrm{M}$ AP5) lead to a modest but non-significant increase in network spiking, indicating that it is possible, yet unlikely, that a partial block of surface NMDARs contributes to the seen increase in network activity. Rather, these data suggest that hNR1 antibody might instead act through specific receptors/neuronal subtypes to disturb $\mathrm{E} / \mathrm{I}$ balance. Indeed, in the presence of hNR1 antibody, cortical networks dramatically increase their bursting activity (Fig. 1J-P) and loose responsiveness to bicuculline (Fig. 3A-F), suggesting that the inhibitory system is likely affected. 
A second puzzling question is why neuronal spiking continues to climb in the presence of hNR1 antibody, when one would expect mechanisms stabilizing network excitability, such as Npas4 signaling (Bloodgood et al. 2013), to counter a hyperactive state. Surprisingly, nuclear Npas4 levels did not increase in the presence of hNR1 antibody (Fig. 2A-D), suggesting that this mechanism became ineffective. Accordingly, normal NMDA-mediated increase in nuclear Npas4 was blocked by addition of hNR1 antibody (Fig. 2E-F). This could be due to a direct effect of hNR1 antibody on NMDARs, as AP5 also prevented the induction of Npas4 after NMDA application (Fig. 2E-F). Together, these data indicate that the hNR1 antibody can interfere with homeostatic mechanisms regulated by NMDAR/Npas4 signaling.

\section{hNR1 antibody impairs the output of cortical inhibitory neurons}

While the depression of NMDAR/Npas4 signaling may explain why hyper-excitability is not scaled down, it does not fully explain why the activity increases in the first place. One possibility is that hNR1 antibody acts on a subset of receptors, synapses or neurons. In the cortex, a correct E/I balance is maintained by GABAergic interneurons orchestrating excitability of pyramidal cells. Our data indicate that cortical GABAergic interneurons are indeed a primary target of hNR1 antibody, specifically altering the output of inhibitory synapses onto excitatory neurons. Consistently, hNR1 antibody treatment decreased action potential-independent, tonic inhibitory drive onto excitatory neurons, measured by frequency of mIPSCs (Fig. 4A-F). This was specific to inhibitory synapses, as mEPSCs frequency remained unchanged (Fig. 4I-P). This is further supported by a decrease in the levels of both VGAT and GAD65 in presynaptic inhibitory boutons formed onto excitatory neurons, but not inhibitory interneurons (Fig. 7).

Importantly, cell-autonomous autaptic recordings confirmed the specific impact of hNR1 antibody on inhibitory neuron function, where it significantly reduced NMDA- and evoked synaptic currents 
exclusively in inhibitory autaptic neurons (Fig. 5). Although reduced NMDA-currents are likely due to receptor internalization, how loss of surface NMDARs translates into reduced inhibitory synaptic transmission remains elusive. Conceivably, reduced NMDAR-mediated $\mathrm{Ca}^{2+}$ influx could trigger changes in transcriptional profiles in these neurons, leading to decreased levels of pre-synaptic proteins (Fig. 7) and ultimately synaptic output (Fig. 5), similarly to networks in which excitation has been removed (Lau and Murthy 2012). However, given that we observed increased somatic calcium signals in all cortical neurons (Fig. 3), whether such mechanisms are involved remains unclear and further RNA sequencing studies are needed to elucidate possible cell-type specific transcriptional changes.

\section{hNR1 antibody shows brain regional specificity}

Remarkably, our data implies that autoantibodies can have different effects in varying brain regions. In cortex, we find that hNR1 specifically impairs cortical inhibitory, but not excitatory, neurons. This contrasts with studies in the hippocampus, showing that these autoantibodies primarily affect excitatory neuron function (Fig. 6, (Kreye et al. 2016; Hughes et al. 2010). This regional specificity indicates that there are limitations with current pre-clinical models, which passively deliver patient CSF/antibodies into ventricles (Planagumà et al. 2016; Taraschenko et al. 2019) or hippocampus (Kersten et al. 2019; Würdemann et al. 2016). While this approach can provide direct antibody access to hippocampal structures, they rarely reach cortical regions (Planagumà et al. 2015). As such, active immunization models which more often report increased sensitivity to seizures (Jones et al. 2019; Wagnon et al. 2020) may provide for a more clinically-relevant distribution of antibodies and thus a better understanding of their region-specific effects.

\section{hNR1 antibody binds specific pools of NMDARs and affects inhibitory synapses}

At present, it is unclear which mechanisms lead to brain regional and/or neuronal type specificity of hNR1 antibody. One possibility could be that the hNR1 antibody binds variably on excitatory versus 
inhibitory neurons. Acute antibody binding studies supports this idea, as hNR1 antibody differentially binds synaptic and extra-synaptic NMDARs on inhibitory vs excitatory cortical neurons (Fig. 8A-B). This is relevant as synaptic and extra-synaptic NMDARs trigger distinct downstream signaling pathways and could therefore contribute to differential effects on each cell type.

An intriguing complementary mechanism could involve local effects of hNR1 antibody on presynaptic NMDARs. Although controversial and debated in the field, pre-synaptic NMDARs within inhibitory synapses have been shown to control neurotransmitter release (Bouvier et al. 2015) and regulate inhibitory drive onto pyramidal neuron in prefrontal cortex (Pafundo et al. 2018; Mathew and Hablitz 2011). Thus, their hypofunction due to hNR1 binding could partially explain the observed decrease in mIPSCs (Fig. 4). Accordingly, we observed that the hNR1 antibody indeed decorates inhibitory synapses (Fig. 8). While it is unclear whether this binding is pre- or post-synaptic, a recent immuno-EM study found that the hNR1 antibody could indeed decorate presynaptic-NMDARs (Wagner et al. 2020). Importantly, studies by Fiszman and colleagues reported that reducing presynaptic-NMDAR signaling decreases the size of pre-synaptic inhibitory boutons, as measured by GAD65 puncta intensity, similar to our observations (Fig. 7). Thus, in addition to more general effects of the hNR1 antibodies on inhibitory interneurons, there is an emerging framework for how these antibodies could also have more selective actions on subsets of inhibitory synapses, which ultimately alter the excitability of cortical networks in patients with NMDAR encephalitis.

\section{Relevance for neuropsychiatric disorders}

Remarkably, NMDAR hypofunction on cortical interneurons has long been implicated to underlie cortical disinhibition and core psychiatric symptoms in schizophrenia (Nakazawa et al. 2012). Genetic deletion of NMDARs in interneurons in vivo produces schizophrenia-like behaviors (Belforte et al. 2010), impairs E/I balance and increases excitability of cortical pyramidal cells (Pafundo et al. 2021). 
Similarly, ketamine impairs mIPSCs, without affecting mEPSCs, and leads to increased pyramidal excitability in prefrontal cortical slices (Y. Zhang, Behrens, and Lisman 2008), which is strikingly similar to our effects after hNR1 antibody treatment (Fig. 1, 3, 4). Our data thus provides evidence that patient-derived autoantibodies can disturb similar pathways and suggest a possible common mechanism across neuropsychiatric disorders.

Importantly, the proposed mechanisms of antibody action are not mutually exclusive and may act in a complementary manner to exert regional changes in synaptic plasticity, subunit-specific transcriptional changes and presynaptic regulation of inhibitory drive to produce complex behavioral and cognitive phenotypes. Together, our data reveals a novel, region-specific mechanism of antiNMDAR antibodies in cortical neurons causing inhibitory dysfunction and network disinhibition.

8




\section{References:}

Arancillo, Marife, Sang-Won Min, Stefan Gerber, Agnieszka Münster-Wandowski, Yuan-Ju Wu, Melissa Herman, Thorsten Trimbuch, Jong-Cheol Rah, Gudrun Ahnert-Hilger, and Dietmar Riedel. 2013. "Titration of Syntaxin1 in Mammalian Synapses Reveals Multiple Roles in Vesicle Docking, Priming, and Release Probability." Journal of Neuroscience 33 (42): 16698714.

Banker, Gary, and K. Goslin. 1988. "Developments in Neuronal Cell Culture." Nature 336 (6195): 185-86.

Belforte, Juan E., Veronika Zsiros, Elyse R. Sklar, Zhihong Jiang, Gu Yu, Yuqing Li, Elizabeth M. Quinlan, and Kazu Nakazawa. 2010. “Postnatal NMDA Receptor Ablation in Corticolimbic Interneurons Confers Schizophrenia-like Phenotypes." Nature Neuroscience 13 (1): 76-83.

Bliss, Tim VP, and Graham L. Collingridge. 1993. “A Synaptic Model of Memory: Long-Term Potentiation in the Hippocampus." Nature 361 (6407): 31-39.

Bloodgood, Brenda L., Nikhil Sharma, Heidi Adlman Browne, Alissa Z. Trepman, and Michael E. Greenberg. 2013. “The Activity-Dependent Transcription Factor NPAS4 Regulates DomainSpecific Inhibition." Nature 503 (7474): 121-25.

Bouvier, Guy, Céline Bidoret, Mariano Casado, and Pierre Paoletti. 2015. “Presynaptic NMDA Receptors: Roles and Rules." Neuroscience 311: 322-40.

Bruijn, Marienke A. A. M. de, Agnes van Sonderen, Marleen H. van Coevorden-Hameete, Anna E. M. Bastiaansen, Marco W. J. Schreurs, Rob P. W. Rouhl, Cees A. van Donselaar, et al. 2019. "Evaluation of Seizure Treatment in Anti-LGI1, Anti-NMDAR, and Anti-GABABR Encephalitis." Neurology 92 (19): e2185-96. https://doi.org/10.1212/WNL.0000000000007475.

Chang, Chia-Ling, Thorsten Trimbuch, Hsiao-Tuan Chao, Julia-Christine Jordan, Melissa A. Herman, and Christian Rosenmund. 2014. "Investigation of Synapse Formation and Function in a Glutamatergic-GABAergic Two-Neuron Microcircuit." Journal of Neuroscience 34 (3): 855-68. 
Chen, Jen-Yung, Sylvain Chauvette, Steven Skorheim, Igor Timofeev, and Maxim Bazhenov. 2012. “Interneuron-Mediated Inhibition Synchronizes Neuronal Activity during Slow Oscillation." The Journal of Physiology 590 (16): 3987-4010.

Dalmau, Josep, and Francesc Graus. 2018. “Antibody-Mediated Encephalitis.” New England Journal of Medicine 378 (9): 840-51.

Dalmau, Josep, Erdem Tüzün, Hai-yan Wu, Jaime Masjuan, Jeffrey E. Rossi, Alfredo Voloschin, Joachim M. Baehring, Haruo Shimazaki, Reiji Koide, and Dale King. 2007. “Paraneoplastic Anti-N-Methyl-D-Aspartate Receptor Encephalitis Associated with Ovarian Teratoma." Annals of Neurology 61 (1): 25-36.

Dana, Hod, Boaz Mohar, Yi Sun, Sujatha Narayan, Andrew Gordus, Jeremy P. Hasseman, Getahun Tsegaye, Graham T. Holt, Amy Hu, and Deepika Walpita. 2016. "Sensitive Red Protein Calcium Indicators for Imaging Neural Activity." Elife 5: e12727.

Emnett, Christine M., Lawrence N. Eisenman, Amanda M. Taylor, Yukitoshi Izumi, Charles F. Zorumski, and Steven Mennerick. 2013. “Indistinguishable Synaptic Pharmacodynamics of the N-Methyl-D-Aspartate Receptor Channel Blockers Memantine and Ketamine." Molecular Pharmacology 84 (6): 935-47.

Fiszman, Mónica L., Andrea Barberis, Congyi Lu, Zhanyan Fu, Ferenc Erdélyi, Gábor Szabó, and Stefano Vicini. 2005. "NMDA Receptors Increase the Size of GABAergic Terminals and Enhance GABA Release." Journal of Neuroscience 25 (8): 2024-31.

Flavell, Steven W., and Michael E. Greenberg. 2008. "Signaling Mechanisms Linking Neuronal Activity to Gene Expression and Plasticity of the Nervous System." Annu. Rev. Neurosci. 31: 563-90.

Gleichman, Amy J., Lynn A. Spruce, Josep Dalmau, Steven H. Seeholzer, and David R. Lynch. 2012. “Anti-NMDA Receptor Encephalitis Antibody Binding Is Dependent on Amino Acid Identity of a Small Region within the GluN1 Amino Terminal Domain." Journal of Neuroscience 32 (32): 11082-94. 
Hardingham, Giles E., and Hilmar Bading. 2003. "The Yin and Yang of NMDA Receptor Signalling." Trends in Neurosciences 26 (2): 81-89. https://doi.org/10.1016/S0166-2236(02)00040-1. Hazan, Hananel, and Noam E. Ziv. 2017. “Closed Loop Experiment Manager (CLEM) -an Open and Inexpensive Solution for Multichannel Electrophysiological Recordings and Closed Loop Experiments." Frontiers in Neuroscience 11: 579.

Hazan, Liran, and Noam E. Ziv. 2020. “Activity Dependent and Independent Determinants of Synaptic Size Diversity." Journal of Neuroscience 40 (14): 2828-48.

Homayoun, Houman, and Bita Moghaddam. 2007a. “NMDA Receptor Hypofunction Produces Opposite Effects on Prefrontal Cortex Interneurons and Pyramidal Neurons." Journal of Neuroscience 27 (43): 11496-500.

- - - 2007b. "NMDA Receptor Hypofunction Produces Opposite Effects on Prefrontal Cortex Interneurons and Pyramidal Neurons." Journal of Neuroscience 27 (43): 11496-500.

Hughes, Ethan G., Xiaoyu Peng, Amy J. Gleichman, Meizan Lai, Lei Zhou, Ryan Tsou, Thomas D. Parsons, David R. Lynch, Josep Dalmau, and Rita J. Balice-Gordon. 2010. “Cellular and Synaptic Mechanisms of Anti-NMDA Receptor Encephalitis." Journal of Neuroscience 30 (17): $5866-75$.

Irani, Sarosh R., Katarzyna Bera, Patrick Waters, Luigi Zuliani, Susan Maxwell, Michael S. Zandi, Manuel A. Friese, Ian Galea, Dimitri M. Kullmann, and David Beeson. 2010. “N-Methyl-DAspartate Antibody Encephalitis: Temporal Progression of Clinical and Paraclinical Observations in a Predominantly Non-Paraneoplastic Disorder of Both Sexes." Brain 133 (6): $1655-67$.

Jantzen, Sabine U., Stefano Ferrea, Claudia Wach, Kim Quasthoff, Sebastian Illes, Dag Scherfeld, Hans-Peter Hartung, Rüdiger J. Seitz, and Marcel Dihné. 2013. “In Vitro Neuronal Network Activity in NMDA Receptor Encephalitis." BMC Neuroscience 14 (1): 1-7. 
Jentsch, J. David, and Robert H. Roth. 1999. "The Neuropsychopharmacology of Phencyclidine: From NMDA Receptor Hypofunction to the Dopamine Hypothesis of Schizophrenia." Neuropsychopharmacology 20 (3): 201-25.

Johnston, Graham AR. 2013. “Advantages of an Antagonist: Bicuculline and Other GABA Antagonists." British Journal of Pharmacology 169 (2): 328-36. https://doi.org/10.1111/bph.12127.

Jones, Brian E., Kenneth R. Tovar, April Goehring, Farzad Jalali-Yazdi, Nana J. Okada, Eric Gouaux, and Gary L. Westbrook. 2019. “Autoimmune Receptor Encephalitis in Mice Induced by Active Immunization with Conformationally Stabilized Holoreceptors." Science Translational Medicine 11 (500).

Kaufman, Maya, Sebastian Reinartz, and Noam E. Ziv. 2014. “Adaptation to Prolonged Neuromodulation in Cortical Cultures: An Invariable Return to Network Synchrony." BMC Biology 12 (1): 1-22.

Kersten, Maxi, Theresa Rabbe, Roman Blome, Katrin Porath, Tina Sellmann, Christian G. Bien, Rüdiger Köhling, and Timo Kirschstein. 2019. “Novel Object Recognition in Rats with NMDAR Dysfunction in CA1 after Stereotactic Injection of Anti-NMDAR Encephalitis Cerebrospinal Fluid." Frontiers in Neurology 10: 586.

Khawaled, Radwan, Andrew Bruening-Wright, John P. Adelman, and J. Maylie. 1999. "Bicuculline Block of Small-Conductance Calcium-Activated Potassium Channels." Pflügers Archiv 438 (3): 314-21. https://doi.org/10.1007/s004240050915.

Koch, Henner, Cristina E. Niturad, Stephan Theiss, Christian G. Bien, Christian Elger, Klaus-Peter Wandinger, Angela Vincent, Michael Malter, Peter Körtvelyessy, and Holger Lerche. 2019. “In Vitro Neuronal Network Activity as a New Functional Diagnostic System to Detect Effects of Cerebrospinal Fluid from Autoimmune Encephalitis Patients." Scientific Reports 9 (1): 1-8. Kreye, Jakob, Nina K. Wenke, Mariya Chayka, Jonas Leubner, Rajagopal Murugan, Nikolaus Maier, Betty Jurek, Lam-Thanh Ly, Doreen Brandl, and Benjamin R. Rost. 2016. “Human 
Cerebrospinal Fluid Monoclonal N-Methyl-D-Aspartate Receptor Autoantibodies Are Sufficient for Encephalitis Pathogenesis." Brain 139 (10): 2641-52.

Krystal, John H., Laurence P. Karper, John P. Seibyl, Glenna K. Freeman, Richard Delaney, J. Douglas Bremner, George R. Heninger, Malcolm B. Bowers, and Dennis S. Charney. 1994. "Subanesthetic Effects of the Noncompetitive NMDA Antagonist, Ketamine, in Humans: Psychotomimetic, Perceptual, Cognitive, and Neuroendocrine Responses." Archives of General Psychiatry 51 (3): 199-214.

Lau, C. Geoffrey, and Venkatesh N. Murthy. 2012. “Activity-Dependent Regulation of Inhibition via GAD67." Journal of Neuroscience 32 (25): 8521-31.

Lois, Carlos, Elizabeth J. Hong, Shirley Pease, Eric J. Brown, and David Baltimore. 2002. “Germline Transmission and Tissue-Specific Expression of Transgenes Delivered by Lentiviral Vectors." Science 295 (5556): 868-72.

Malviya, Manish, Sumanta Barman, Kristin S. Golombeck, Jesús Planagumà, Francesco Mannara, Nathalie Strutz-Seebohm, Claudia Wrzos, Fatih Demir, Christine Baksmeier, and Julia Steckel. 2017. “NMDAR Encephalitis: Passive Transfer from Man to Mouse by a Recombinant Antibody." Annals of Clinical and Translational Neurology 4 (11): 768-83.

Manto, Mario, Josep Dalmau, Adrien Didelot, Véronique Rogemond, and Jérôme Honnorat. 2010. “In Vivo Effects of Antibodies from Patients with Anti-NMDA Receptor Encephalitis: Further Evidence of Synaptic Glutamatergic Dysfunction." Orphanet Journal of Rare Diseases 5 (1): $1-12$.

Mathew, Seena S., and John J. Hablitz. 2011. “Presynaptic NMDA Receptors Mediate IPSC Potentiation at GABAergic Synapses in Developing Rat Neocortex." PloS One 6 (2): e17311.

Meberg, Peter J., and Matthew W. Miller. 2003. "Culturing Hippocampal and Cortical Neurons.” Methods Cell Biol 71 (2): 111-27.

Mikasova, Lenka, Pierre De Rossi, Delphine Bouchet, François Georges, Véronique Rogemond, Adrien Didelot, Claire Meissirel, Jérôme Honnorat, and Laurent Groc. 2012. “Disrupted Surface 
(5): 1606-21.

Minerbi, Amir, Roni Kahana, Larissa Goldfeld, Maya Kaufman, Shimon Marom, and Noam E. Ziv. 2009. “Long-Term Relationships between Synaptic Tenacity, Synaptic Remodeling, and Network Activity." PLoS Biol 7 (6): e1000136.

Moscato, Emilia H., Xiaoyu Peng, Ankit Jain, Thomas D. Parsons, Josep Dalmau, and Rita J. BaliceGordon. 2014. “Acute Mechanisms Underlying Antibody Effects in Anti-N-Methyl-DAspartate Receptor Encephalitis." Annals of Neurology 76 (1): 108-19.

Nakazawa, Kazu, Veronika Zsiros, Zhihong Jiang, Kazuhito Nakao, Stefan Kolata, Shuqin Zhang, and Juan E. Belforte. 2012. "GABAergic Interneuron Origin of Schizophrenia Pathophysiology." Neuropharmacology 62 (3): 1574-83.

Nicoll, Roger A. 2017. “A Brief History of Long-Term Potentiation.” Neuron 93 (2): 281-90. https://doi.org/10.1016/j.neuron.2016.12.015.

Pafundo, Diego E., Takeaki Miyamae, David A. Lewis, and Guillermo Gonzalez-Burgos. 2018. “Presynaptic Effects of N-Methyl-D-Aspartate Receptors Enhance Parvalbumin CellMediated Inhibition of Pyramidal Cells in Mouse Prefrontal Cortex." Biological Psychiatry 84 (6): 460-70.

Pafundo, Diego E., Carlos A. Pretell Annan, Nicolas M. Fulginiti, and Juan E. Belforte. 2021. “Early NMDA Receptor Ablation in Interneurons Causes an Activity-Dependent E/I Imbalance in Vivo in Prefrontal Cortex Pyramidal Neurons of a Mouse Model Useful for the Study of Schizophrenia." Schizophrenia Bulletin.

Planagumà, Jesús, Holger Haselmann, Francesco Mannara, Mar Petit-Pedrol, Benedikt Grünewald, Esther Aguilar, Luise Röpke, Elena Martín-García, Maarten J. Titulaer, and Pablo Jercog. 2016. “Ephrin-B2 Prevents N-Methyl-D-Aspartate Receptor Antibody Effects on Memory and Neuroplasticity." Annals of Neurology 80 (3): 388-400. 
Planagumà, Jesús, Frank Leypoldt, Francesco Mannara, Javier Gutiérrez-Cuesta, Elena Martín-García, Esther Aguilar, Maarten J. Titulaer, et al. 2015. “Human N-Methyl D-Aspartate Receptor Antibodies Alter Memory and Behaviour in Mice." Brain 138 (1): 94-109. https://doi.org/10.1093/brain/awu310.

Spiegel, Ivo, Alan R. Mardinly, Harrison W. Gabel, Jeremy E. Bazinet, Cameron H. Couch, Christopher P. Tzeng, David A. Harmin, and Michael E. Greenberg. 2014. “Npas4 Regulates ExcitatoryInhibitory Balance within Neural Circuits through Cell-Type-Specific Gene Programs." Cell 157 (5): 1216-29.

Tamamaki, Nobuaki, Yuchio Yanagawa, Ryohei Tomioka, Jun-Ichi Miyazaki, Kunihiko Obata, and Takeshi Kaneko. 2003. “Green Fluorescent Protein Expression and Colocalization with Calretinin, Parvalbumin, and Somatostatin in the GAD67-GFP Knock-in Mouse." Journal of Comparative Neurology 467 (1): 60-79.

Taraschenko, Olga, Howard S. Fox, Sean J. Pittock, Anastasia Zekeridou, Maftuna Gafurova, Ember Eldridge, Jinxu Liu, Shashank M. Dravid, and Raymond Dingledine. 2019. “A Mouse Model of Seizures in Anti-N-Methyl-d-Aspartate Receptor Encephalitis." Epilepsia 60 (3): 452-63.

Teppola, Heidi, Jugoslava Aćimović, and Marja-Leena Linne. 2019. “Unique Features of Network Bursts Emerge from the Complex Interplay of Excitatory and Inhibitory Receptors in Rat Neocortical Networks." Frontiers in Cellular Neuroscience 13: 377.

Teppola, Heidi, S. Okujeni, M.-L. Linne, and U. Egert. 2018. “AMPA, NMDA and GABAA Receptor Mediated Network Burst Dynamics in Cortical Cultures in Vitro." ArXiv Preprint ArXiv:1802.00217.

Titulaer, Maarten J, Lindsey McCracken, Iñigo Gabilondo, Thaís Armangué, Carol Glaser, Takahiro lizuka, Lawrence S Honig, et al. 2013. “Treatment and Prognostic Factors for Long-Term Outcome in Patients with Anti-NMDA Receptor Encephalitis: An Observational Cohort Study." The Lancet Neurology 12 (2): 157-65. https://doi.org/10.1016/S14744422(12)70310-1. 
Uhlhaas, Peter J., and Wolf Singer. 2010. “Abnormal Neural Oscillations and Synchrony in Schizophrenia." Nature Reviews Neuroscience 11 (2): 100-113.

Wagner, Franziska, Angelika Goertzen, Orsolya Kiraly, Gregor Laube, Jakob Kreye, Otto W. Witte, Harald Prüss, and Rüdiger W. Veh. 2020. “Detailed Morphological Analysis of Rat Hippocampi Treated with CSF Autoantibodies from Patients with Anti-NMDAR Encephalitis Discloses Two Distinct Types of Immunostaining Patterns." Brain Research 1747: 147033.

Wagnon, Isabelle, Pauline Hélie, Isabelle Bardou, Caroline Regnauld, Léonie Lesec, Jerôme Leprince, Mikaël Naveau, Barbara Delaunay, Olivier Toutirais, and Brigitte Lemauff. 2020. “Autoimmune Encephalitis Mediated by B-Cell Response against N-Methyl-d-Aspartate Receptor." Brain 143 (10): 2957-72.

Wardemann, Hedda, Sergey Yurasov, Anne Schaefer, James W. Young, Eric Meffre, and Michel C. Nussenzweig. 2003. “Predominant Autoantibody Production by Early Human B Cell Precursors." Science 301 (5638): 1374-77.

Wright, Sukhvir, Kevan Hashemi, Lukasz Stasiak, Julian Bartram, Bethan Lang, Angela Vincent, and A. Louise Upton. 2015. “Epileptogenic Effects of NMDAR Antibodies in a Passive Transfer Mouse Model." Brain 138 (11): 3159-67.

Würdemann, Till, Maxi Kersten, Tursonjan Tokay, Xiati Guli, Maria Kober, Marco Rohde, Katrin Porath, Tina Sellmann, Christian G. Bien, and Rüdiger Köhling. 2016. “Stereotactic Injection of Cerebrospinal Fluid from Anti-NMDA Receptor Encephalitis into Rat Dentate Gyrus Impairs NMDA Receptor Function." Brain Research 1633: 10-18.

Zhang, Qing, Keiko Tanaka, Peng Sun, Michiyo Nakata, Ryo Yamamoto, Kenji Sakimura, Makoto Matsui, and Nobuo Kato. 2012. "Suppression of Synaptic Plasticity by Cerebrospinal Fluid from Anti-NMDA Receptor Encephalitis Patients." Neurobiology of Disease 45 (1): 610-15.

Zhang, Yuchun, M. Margarita Behrens, and John E. Lisman. 2008a. “Prolonged Exposure to NMDAR Antagonist Suppresses Inhibitory Synaptic Transmission in Prefrontal Cortex." Journal of Neurophysiology 100 (2): 959-65. 

2008b. "Prolonged Exposure to NMDAR Antagonist Suppresses Inhibitory Synaptic Transmission in Prefrontal Cortex." Journal of Neurophysiology 100 (2): 959-65.

954 https://doi.org/10.1152/jn.00079.2008.

955

956

958 
Figure 1. hNR1 antibody increases spiking and bursting activity of cortical cultures. (A, I) Experimental design of multi-electrode array (MEA) experiments. Baseline activity of cortical networks was recorded for $15 \mathrm{~h}$, followed by addition of $1 \mathrm{ug} / \mathrm{ml}$ of hNR1 antibody, control antibody or $1 \mu \mathrm{M}$ of AP5 and $24 \mathrm{~h}$ recording of activity in the presence of antibodies $/ 1 \mu \mathrm{M}$ AP5. After $24 \mathrm{~h}$ of treatment, saturating concentration of AP5 (50 $\mu \mathrm{M})$ was added to the network and another $6 \mathrm{~h}$ of activity were recorded. (B) Normalized spike rate of neuronal networks. Each data point represents a number of spikes recorded within a 10 min interval from all 59 electrodes and normalized to the last $3 \mathrm{~h}$ of baseline activity. Addition of hNR1 antibody (green data points), and to a much lesser degree control (ctrl, mGO53) antibody (grey data points) or AP5 at its IC50 concentration $(1 \mu \mathrm{M})$ (yellow data points), increase spiking of the network. hNR1 $=5$ independent experiments, ctrl $=5$ independent experiments, AP5 $=3$ independent experiments. Error bars indicate SEM. (C-E) Within-dish comparison of normalized spike rate of the same network at the end of baseline recording (1h) and after $24 \mathrm{~h}$ of treatment $(1 \mathrm{~h})$ tested by paired t-test. $(\mathrm{C})$ baseline $=1.01 \pm 0.01$, after $24 \mathrm{~h}$ ctrl $=1.72 \pm 0.27,5, p=0.07$; (D) baseline $=1,12 \pm 0.47$, after $24 \mathrm{~h}$ hNR1 $=3.02 \pm 0.47, \mathrm{p}=0.02 ;(\mathrm{E})$ baseline $=1.02 \pm 0.01$, after $24 \mathrm{~h} 1 \mu \mathrm{M}$ AP5 $=$ $1.43 \pm 0.13, p=0.09$. (F-H) Percentage increase in normalized spike rate at the end (10 $\mathrm{min})$ or beginning (10 $\min$ ) of respective treatment. Each data point represents individual network (MEA dish). Percentage increase in spike rate: $(\mathbf{F})$ at the end of baseline compared to end of antibody treatment, $\mathrm{ctrl}=71.71 \% \pm 27.46$; $\mathrm{hNR} 1=$ $200.5 \% \pm 42.96,1 \mu \mathrm{M} \mathrm{AP5}=42.4 \% \pm 10.76$, ctrl vs hNR1 $p=0.045$, hNR1 vs $1 \mu \mathrm{M}$ AP5 $p=0.034 ;(G)$ at the end of antibody treatment compared to beginning of $50 \mu \mathrm{M} \mathrm{AP5}$, ctrl $=-94.96 \% \pm 5.03 ; \mathrm{hNR} 1=-41.69 \% \pm 15.88 ; 1$ $\mu \mathrm{M}$ AP5 $=-86.51 \% \pm 1.33, \mathrm{ctrl}$ vs $\mathrm{hNR} 1 \mathrm{p}=0.085 ;(\mathrm{H})$ at the end of antibody treatment compared to recovery $6 \mathrm{~h}$ after addition of $50 \mu \mathrm{M} \mathrm{AP5}, \mathrm{ctrl}=61.34 \% \pm 10.65 ; \mathrm{hNR} 1=92.65 \% \pm 8.8 ; 1 \mu \mathrm{M}$ AP5 $=45.04 \% \pm 4.88, \mathrm{hNR} 1$ vs $1 \mu \mathrm{M}$ AP5 $p=0.038$. (J) Normalized burst rate of neuronal networks. Each data point represents a number of bursts recorded within a $10 \mathrm{~min}$ interval, normalized to the last $3 \mathrm{~h}$ of baseline. Network bursting increases in the presence of hNR1 antibody, but not of control antibody nor $1 \mu \mathrm{M}$ AP5. (K-M) Within-dish comparison of normalized burst rates of the same networks at the end of baseline recording (1h) and after $24 \mathrm{~h}$ of treatment (1h) tested by paired t-test. $(\mathrm{K})$ baseline $=1 \pm 0.03$; after $24 \mathrm{~h}$ ctrl $=1.05 \pm 0.12, p=0.72 ;(\mathrm{L})$ baseline $=1.04 \pm$ $0.03 ;$ after $24 \mathrm{~h} h N R 1=3.22 \pm 0.63, p=0.027 ;(\mathrm{M})$ baseline $=1 \pm 0.04 ;$ after $24 \mathrm{~h} 1 \mu \mathrm{M} \mathrm{AP5}=1.36 \pm 0.2, \mathrm{p}=0.2$. (N-P) Percentage increase in normalized burst rate at the end $(10 \mathrm{~min})$ or beginning $(10 \mathrm{~min})$ of respective treatments. Percentage increase in burst rate: (N) at the end of baseline compared to end of antibody 
999

1000

100

1002

1003

1004

1005

1006

1007

1008

1009

1010

1011

1012

1013

1014

1015

1016

1017

1018

1019

treatment, $\mathrm{ctrl}=23.31 \% \pm 19.47 ; \mathrm{hNR} 1=247.5 \% \pm 66.75 ; 1 \mu \mathrm{M} \mathrm{AP5}=30.14 \% \pm 25.57, \mathrm{ctrl}$ vs hNR1 $\mathrm{p}=0.01$, hNR1 vs $1 \mu \mathrm{M}$ AP5 $\mathrm{p}=0.03$; (0) end of antibody treatment compared to beginning of $50 \mu \mathrm{M}$ AP5, ctrl = $70.46 \% \pm 9.9 ; \mathrm{hNR} 1=-36.58 \% \pm 25.24 ; 1 \mu \mathrm{M} \mathrm{AP5}=-26.05 \% \pm 25.24 ;(\mathrm{P})$ end of antibody treatment compared to recovery $6 \mathrm{~h}$ after addition of $50 \mu \mathrm{M} \mathrm{AP5}, \mathrm{ctrl}=147 \% \pm 64.39 ; \mathrm{hNR} 1=149.6 \% \pm 16.73 ; 1 \mu \mathrm{M} \mathrm{AP5}=143 \% \pm$ 26.56. Error bars indicate SEM. Paired t-test (C-E, K-M) or ANOVA Tukey's multiple-comparison test (F-H, N-P) were used to evaluate statistical significance. ${ }^{*} p<0.05$. were used to evaluate statistical significance. *p<0.05. 
Figure 2. hNR1 antibody dysregulates NMDA-induced expression of Npas4, a transcription factor regulating

1021 network activity. (A-D) Cortical neuronal cultures 14-16 DIV were incubated with $1 \mu \mathrm{g} / \mathrm{ml}$ of hNR1 antibody for $2 \mathrm{~h}, 4 \mathrm{~h}$ or $24 \mathrm{~h}$, then fixed and stained with anti-Npas 4 antibody to measure intensity of somatic Npas4 expression. Neither short-term (2h, 4h, A) nor long-term (24h, C) treatment with hNR1 induced somatic Npas4 expression. (B, D) Normalized intensity of somatic Npas4 signal from 3 independent experiments: (B) UT = $1 \pm$ $0.03, \mathrm{n}=218$ neurons; $2 \mathrm{~h}$ hNR1 $=0.97 \pm 0.04, \mathrm{n}=133$ neurons; $4 \mathrm{~h} h N R 1=1.08 \pm 0.03, \mathrm{n}=168$ neurons. (D) $=1 \pm 0.04, \mathrm{n}=280$ neurons; $24 \mathrm{~h} \mathrm{hNR} 1=0.98 \pm 0.03, \mathrm{n}=183$ neurons. (E) Stimulation of neurons with $2 \mu \mathrm{M}$ NMDA for $2 \mathrm{~h}$ induces activity-driven increase of Npas 4 expression compared to untreated condition. $6 \mathrm{~h}$ or $24 \mathrm{~h}$ pretreatment with hNR1 antibody $(1 \mu \mathrm{g} / \mathrm{ml})$, as well as $3 \mathrm{~h}$ pretreatment with NMDAR antagonist AP5 (50 $\mu \mathrm{M})$,

1029 blocks NMDA-induced expression of Npas4. (F) Normalized intensity of somatic Npas4 signal from 3 independent experiments: UT $=1 \pm 0.03, \mathrm{n}=235$ neurons; NMDA $=2.21 \pm 0.17, \mathrm{n}=197$ neurons; $6 \mathrm{~h}$ NR1 + $\mathrm{NMDA}=1.28 \pm 0.04, \mathrm{n}=172$ neurons; $24 \mathrm{~h} \mathrm{hNR} 1-\mathrm{NMDA}=1.13 \pm 0.03, \mathrm{n}=179$ neurons; $3 \mathrm{~h}$ AP5 $+\mathrm{NMDA}=$ 
1045

1046

1047

1048

1049

1050

1051

1052

1053

1054

1055

1056

1057

1058

1059

1060

1061

1062

1063

1064

1065

1066

1067

1068

1069

Figure 3. Calcium imaging reveals that networks treated with hNR1 antibody cannot be further disinhibited by bicuculline. (A-C) Representative raster plots of calcium events recorded from neuronal somas (neuron ID on the $y$ axis) expressing RCamp in untreated condition ( $A$, upper panel), after $24 \mathrm{~h}$ of control antibody (B, upper panel) or $24 \mathrm{~h}$ of hNR1 antibody (C, upper panel) treatment. Lower panels in (A-C) show representative raster plots of calcium events of the same neurons as in upper panels following addition of bicuculline (30 $\mu \mathrm{M})$ at the end of respective treatment. (D) Frequency of somatic calcium events in untreated condition, after $24 \mathrm{~h}$ of hNR1 or control antibodies treatment (upper panels in A-C); UT $=10.89 \pm 0.31, \mathrm{n}=366$ cells, ctrl $=12.55 \pm$ $0.43, \mathrm{n}=277$ cells, hNR1 $=14.99 \pm 0.37, \mathrm{n}=391$ cells, 6 independent experiments, ${ }^{* *} \mathrm{p}=0.007, * * * * \mathrm{p}<$ 0.0001. (E-G) Within-cell comparison of the frequency of calcium events in the same cells at the end of $24 \mathrm{~h}$ treatment and after addition of bicuculline tested by paired t-test from 3 independent experiments reveals that bicuculline disinhibits the networks in untreated condition (D) and after control antibodies treatment (E) but not after hNR1 (F) treatment. Quantification of calcium events: (E) UT $=4.83 \pm 0,51$, UT + Bic $=10.87 \pm 0,81$, mean of difference $=6.05 \pm 0.75, \mathrm{n}=47$ neurons, $\mathrm{p}<0.0001 ;(\mathbf{F}) 24 \mathrm{~h}$ ctrl $=6.84 \pm 0.0 .74,24 \mathrm{~h}$ ctrl + Bic $=8.61 \pm$ 0,59 , mean of difference $=1.76 \pm 0.82, \mathrm{n}=80$ neurons, $\mathrm{p}=0.035 ;$ (G) $24 \mathrm{~h} \mathrm{hNR} 1=9.88 \pm 0.88,24 \mathrm{~h} \mathrm{hNR} 1+\mathrm{Bic}=$ $10.24 \pm 0.45$, mean of difference $=0.57 \pm 0.9, n=62$ neurons, $p=0.53$. Error bars indicate SEM. ANOVA Tukey's multiple-comparison test (D) and paired t-test (E-G) were used to evaluate statistical significance. ${ }^{*} \mathrm{p}<0.05, * * p<0.01, * * * * p<0.0001$, ns $=$ not significant. 
1070

1071

1072

1073

1074

1075

1076

1077

1078

1079

1080

1081

1082

1083

1084

1085

1086

1087

1088

1089

1090

1091

1092

1093

1094

1095

1096

1097

Figure 4. hNR1 antibodies decrease inhibitory drive onto excitatory neurons in cortical circuits, but do not affect excitatory drive. (A-H) Inhibitory drive onto excitatory neurons was measured by miniature inhibitory post-synaptic currents (mIPSCs) recorded from excitatory neurons in cortical neuronal networks. (A) Schematic model of a simplified neuronal network composed of excitatory pyramidal neurons (red) and inhibitory neurons (blue), forming different types of synapses onto each other. Inhibitory synapse onto excitatory neuron is marked with a circle as a synapse of interest measured in this experiment. (B) mIPSCs were recorded from excitatory neurons identified by expression of mKate2 expressed under excitatory neuron CamKII promoter. Left panel: BF image of representative cortical neuronal network, right panel: excitatory neuron identified by mKate2 fluorescent signal. (C) Representative traces of mIPSCs recorded in a whole-cell patch-clamp configuration from excitatory neurons after $24 \mathrm{~h}$ of treatment with control (upper panel) or hNR1 (lower panel) antibodies. Treatment with hNR1 antibody decreases frequency of mIPSCs onto excitatory neurons (D) and has tendency to decrease their amplitude (E), without affecting their charge (F). (D-F) Quantification of mIPSCs parameters from 3 independent experiments, $\operatorname{ctrl} n=33$ neurons, hNR1 $n=34$ neurons: (D) mIPSCs frequency: $\mathrm{ctrl}=6.42 \pm 0.37, \mathrm{hNR} 1=4.94 \pm 0.4, \mathrm{p}=0.009 ;$ (E) mIPSCs amplitude: $\mathrm{ctrl}=46.89 \pm 2.89, \mathrm{hNR} 1=40.01 \pm 2.25$, $p=0.06 ;$ (F) mIPSCs charge: $c t r l=606.5 \pm 29.72, h N R 1=610 \pm 36.37$. (G-H) hNR1 antibody treatment does not affect levels of GABA receptors on the post-synaptic excitatory neurons from which mIPSCs were recorded. (G) Representative traces of GABA currents recorded from excitatory neurons, evoked by 1 s pulse application of 5 $\mu \mathrm{M}$ GABA. (H) Quantification of GABA current amplitude presented in (F): ctrl $=6.84 \pm 1.1, \mathrm{n}=23$ neurons, hNR1 $=7.6 \pm 1.04, \mathrm{n}=7.6 \pm 1.04, \mathrm{n}=25$ neurons. (I-P) hNR1 antibody does not affect excitatory miniature transmission (mEPSCs) onto neither inhibitory (I-L) nor excitatory (M-P) neurons. (I, M) Representative traces of mEPSCs recorded from inhibitory (I) or excitatory (M) neurons. Quantification of mEPSCs parameters from 3 independent experiments: ( $\mathrm{J})$ mEPSCs frequency: $\operatorname{ctrl}=9.83 \pm 1.41, \mathrm{n}=27$ neurons, $\mathrm{hNR} 1=9.17 \pm 1.2, \mathrm{n}=26$ neurons; (K) mEPSCs amplitude: $\mathrm{ctrl}=36.7 \pm 2.71, \mathrm{n}=26$ neurons, $\mathrm{hNR} 1=34.41 \pm 2.68, \mathrm{n}=26$ neurons; (L) mEPSCs charge: $97.83 \pm 5.47, \mathrm{n}=26$ neurons, $\mathrm{hNR} 1=91.52 \pm 4.02, \mathrm{n}=24$ neurons; (N) mEPSCs frequency: ctrl $=1.32 \pm 0.23, \mathrm{n}=28$ neurons, $\mathrm{hNR} 1=1.98 \pm 0.21, \mathrm{n}=28$ neurons; $(\mathbf{0})$ mEPSCs amplitude: $\mathrm{ctrl}=20.09 \pm 0.93, \mathrm{n}$ $=25$ neurons, $h N R 1=19.84 \pm 0.92, \mathrm{n}=28$ neurons; $(\mathrm{P})$ mEPSCs charge: $\operatorname{ctrl}=66.76 \pm 2.96, \mathrm{n}=23$ neurons, hNR1 $=64.86 \pm 3.22, \mathrm{~h}=24$ neurons). Error bars indicate SEM. Unpaired t-test was used to evaluate statistical significance. ${ }^{* *} p<0.01$. 
Figure 5. hNR1 antibody impairs function of cortical inhibitory, but not excitatory neurons, in a cellautonomous manner. (A) Example image of a single cortical neuron grown on an astrocytic island in autaptic culture and a schematic representation of a patch pipette approaching the neuron. (B-D) Lower panels: representative traces of (B) whole-cell NMDA currents evoked by 1s bath application of NMDA (10 $\mu M),(C)$ evoked excitatory post-synaptic currents (EPSCs), (D) evoked synaptic NMDA currents in excitatory neurons after $24 \mathrm{~h}$ treatment with control or hNR1 antibodies. Upper panels: Normalized current amplitudes from 4 independent experiments on excitatory neurons (B): $\operatorname{ctrl}=1 \pm 0.08, \mathrm{n}=32$ neurons, $h N R 1=1.01 \pm 0.11, \mathrm{n}=32$ neurons; (C): $\operatorname{ctrl}=1 \pm 0.13, \mathrm{n}=33$ neurons, hNR1 $=1.31 \pm 0.19, \mathrm{n}=31$ neurons; and (D): $\operatorname{ctrl}=1 \pm 0.12, \mathrm{n}=29$ neurons, hNR1 $=1.38 \pm 0.23, \mathrm{n}=22$ neurons. $(\mathrm{E}-\mathrm{G})$ Lower panels: representative traces of $(\mathrm{E})$ whole-cell NMDA currents, (F) evoked inhibitory post-synaptic currents (IPSCs), (G) whole-cell GABA currents evoked by 1s bath application of GABA ( $5 \mu \mathrm{M})$ of inhibitory neurons after $24 \mathrm{~h}$ treatment with control or hNR1 antibodies. Upper panels: normalized current amplitudes from 4 independent experiments: (E) $\operatorname{ctrl}=1 \pm 0.1, n=31$ neurons, $\mathrm{hNR} 1=0.71 \pm 0.07, \mathrm{n}=36$ neurons, $\mathrm{p}=0.018 ;(\mathrm{F}) \mathrm{ctrl}=1 \pm 0.12, \mathrm{n}=35$ neurons, $\mathrm{hNR} 1=0.59 \pm 0.11, \mathrm{n}=37$ neurons, $\mathrm{p}=0.014$; $(\mathrm{G}) \mathrm{ctrl}=1 \pm 0.1, \mathrm{n}=25$ neurons, hNR1 $=0.81 \pm 0.11, \mathrm{n}=26$ neurons. $(\mathrm{H})$ Correlation between normalized IPSC and NMDA current amplitudes of individual inhibitory neurons. Linear regression: $\mathrm{R}^{2}$ $=0.132, p=0.029$. (I-L) Analysis of mIPSCs recorded from autaptic inhibitory neurons after $24 \mathrm{~h}$ of control or hNR1 antibodies treatment, 4 independent experiments. (I) Representative traces of mIPSCs. (J) mIPSCs frequency: $\operatorname{ctrl}=1.66 \pm 0.37, \mathrm{n}=31$ neurons, $\mathrm{hNR} 1=1.08 \pm 0.21, \mathrm{n}=35$ neurons. $(\mathrm{K})$ mIPSCs amplitude: $\mathrm{ctrl}=$ $43.87 \pm 2.81, \mathrm{n}=29$ neurons, hNR1 $=30.72 \pm 2.46, \mathrm{n}=32$ neurons, $\mathrm{p}=0.0008$. (L) mIPSCs charge: $\operatorname{ctrl}=789.7 \pm$ 76.58, $\mathrm{n}=29$ neurons, hNR1 $=551.9 \pm 58.94, \mathrm{n}=32$ neurons, $\mathrm{p}=0.016$. Error bars indicate SEM. Unpaired $\mathrm{t}-$ 
Figure 6. hNR1 antibody impairs NMDA currents of excitatory hippocampal neurons. In hippocampal autaptic

1125 cultures, $24 \mathrm{~h}$ treatment with hNR1 antibody $(1 \mu \mathrm{g} / \mathrm{ml})$ selectively decreases whole-cell (A) and synaptic (E) NMDA currents of excitatory neurons. (A-C) Whole-cell receptor currents were evoked by 1 s bath application of (A) NMDA $(10 \mu \mathrm{M})$, (B) GABA $(30 \mu \mathrm{M})$ or (C) kainate $(20 \mu \mathrm{M})$. Quantification of normalized current amplitudes from 3 independent experiments: (A) NMDA currents: ctrl $=1 \pm 0.08, n=30$ neurons, hNR1 $=0.64 \pm 0.06, n=$ 23 neurons, $p=0.0008$; (B) GABA currents: $c t r l=1 \pm 0.08, n=31$ neurons, $h N R 1=0.94 \pm 0.09 . n=22$ neurons;

1130 (C) kainate currents: ctrl $=1 \pm 0.14, \mathrm{n}=21$ neurons, hNR1 $=0.83 \pm 0.15, \mathrm{n}=16$ neurons. (D-E) Synaptic responses were evoked by a brief somatic depolarization of neurons from $-70 \mathrm{mV}$ to $0 \mathrm{mV}$ for $2 \mathrm{~ms}$, synaptic NMDA currents were recorded in the presence of $0 \mathrm{mM} \mathrm{Mg2+,} 10 \mu \mathrm{M}$ glycine and $10 \mu \mathrm{M}$ NBQX. Quantification of normalized current amplitudes from 3 independent experiments: (D) EPSCs: ctrl $=1 \pm 0.13, n=31$ neurons, $\mathrm{hNR} 1=0.99 \pm 0.14, \mathrm{n}=23$ neurons; $(\mathrm{E})$ synaptic NMDA: ctrl $=1 \pm 0.15, \mathrm{n}=26$ neurons, $\mathrm{hNR} 1=0.58 \pm 0.12, \mathrm{n}=$ 20 neurons, $p=0.039$. Error bars indicate SEM. Unpaired t-test was used to evaluate statistical significance. $* p<0.05, * * * p<0.001$. 
Figure 7. hNR1 antibodies decrease levels of inhibitory pre-synaptic proteins VGAT and GAD65 in inhibitory-

1151 to-excitatory neuron synapses. (A) Schematic model showing investigated inhibitory synapses and summarizing the finding: decreased levels of GAD65 and VGAT at inhibitory synapses onto excitatory (yellow circle), but not inhibitory (green circle), neurons. (B-C) Representative images of cortical excitatory (B) and

1154 inhibitory (C) neurons identified by staining with antibodies against GFP. Only proximal dendrites close to soma, which are easily identified as GFP-positive or GFP-negative, were chosen for ROI selection (boxes). (D, G) Images of ROIs of cortical excitatory and inhibitory neurons which were fixed and stained with antibodies against GFP, MAP2 and either VGAT (D) or GAD65 (G), in untreated condition or after $6 \mathrm{~h}$ or $24 \mathrm{~h}$ of hNR1 antibodies treatment. (E-F) Normalized intensity of VGAT puncta along dendrites from 3 independent experiments: (E) on excitatory neurons: UT $=1 \pm 0.05, n=56$ ROls, $24 \mathrm{~h} h N R 1=0.82 \pm 0.04, n=55$ ROIs, $p=$ 0.005. (F) on inhibitory neurons: UT $=1 \pm 0.04, \mathrm{n}=47 \mathrm{ROIs,} 24 \mathrm{~h} h N R 1=0.96 \pm 0.03, \mathrm{n}=57$ ROls. (H-I) Normalized intensity of GAD65 puncta along dendrites from 3 independent experiments: $(H)$ on excitatory neurons: UT $=1 \pm 0.03, \mathrm{n}=64$ ROIs, $6 \mathrm{~h} h N R 1=0.89 \pm 0.02, \mathrm{n}=59$ ROIs, $24 \mathrm{~h} \mathrm{hNR} 1=0.95 \pm 0.02, \mathrm{n}=67 \mathrm{ROIs}, \mathrm{p}$ $=0.01$; (I) on inhibitory neurons: UT $=1 \pm 0.02, \mathrm{n}=64$ ROIs, 6h hNR1 $=0.94 \pm 0.03, \mathrm{n}=53$ ROIs, $24 \mathrm{~h}$ hNR1 $=$ $0.96 \pm 0.03, n=58$ ROIs. Error bars indicate SEM. Scale bar $=10 \mu \mathrm{m}$. Paired t-test $(E-F)$ and ANOVA Tukey's multiple-comparison test $(\mathrm{H}-\mathrm{I})$ were used to evaluate statistical significance. ${ }^{*} \mathrm{p}<0.05,{ }^{*} \mathrm{p}<0.01$. 
1175

1176

1177

1178

1179

1180

1181

1182

1183

1184

1185

1186

1187

1188

1189

1190

1191

1192

1193

1194

1195

1196

1197

1198

1199

Fig 8. hNR1 antibody binds inhibitory synapses, preferentially on excitatory neurons. (A) Images of cortical neurons which were live stained with hNR1 antibody to detect acute binding pattern of the antibodies, and then fixed and co-stained for excitatory synaptic marker VGLUT1. Degree of colocalization of hNR1 antibody puncta and VGLUT1 puncta was used as a measure of excitatory synaptic or extra-synaptic staining. (B) Percentage of hNR1 antibody puncta positive for VGLUT1 (synaptic staining) per ROI: excitatory neurons = $47.28 \pm 4.53, n=28$ ROIs, inhibitory neurons $=69.75 \pm 4.19, n=35$ ROIs, 2 independent experiments, $p=$ 0.006. (C) Images of cortical neurons live stained with hNR1 antibodies for acute hNR1 antibodies binding pattern, which were then fixed and co-stained with inhibitory synaptic marker gephyrin. Degree of colocalization between hNR1 antibody puncta and gephyrin puncta was used to assess inhibitory synaptic binding of the antibodies. Arrowheads indicate overlap between hNR1 and gephyrin. (D) Lower panel: 3D (z stack) volume images of individual inhibitory synapses from images in the upper panel at the level of yellow line, representing cross-section through 12 planes of z-stack. (E) Percentage of gephyrin puncta positive for hNR1 antibodies from 3 independent experiments: excitatory neurons $=30.84 \pm 2.05, n=53$ ROls, inhibitory neurons $=11.17 \pm 1.52, n=48$ ROls, $p<0.0001$. (F) Schematic summarizing findings from these experiments: hNR1 antibody bind within inhibitory synapses, preferentially onto excitatory neurons. Scale bar in A, C = 10 $\mu \mathrm{m}$; in $\mathrm{D}=5 \mu \mathrm{m}$. Error bars indicate SEM. Unpaired t-test was used to evaluate statistical significance. ${ }^{*} p<0.05$, $* * p<0.01, * * * * p<0.0001$. 
1200

1201

1202

1203

1204

1205

1206

1207

1208

Fig 9. Speculative model of effects of hNR1 antibody on cortical inhibitory neuron and network function. In cortical neuronal cultures, hNR1 antibody does not affect NMDA currents (A) or synaptic transmission (B) of excitatory neurons, yet it selectively decreases NMDA currents on inhibitory cells (C). Antibody binding further impairs inhibitory synaptic transmission (D, E) and decreases levels of GABA producing pre-synaptic proteins (F, G), specifically in inhibitory-to-excitatory neuron synapses (yellow circle). Such reduced synaptic inhibitory output could result from altered transcriptional profiles of inhibitory neurons and/or local dysfunction of presynaptic NMDARs within these inhibitory synapses $(\mathbf{H})$, mechanisms yet to be explored. This reduced inhibitory function disinhibits activity of excitatory neurons and the network as a whole (I, J). 

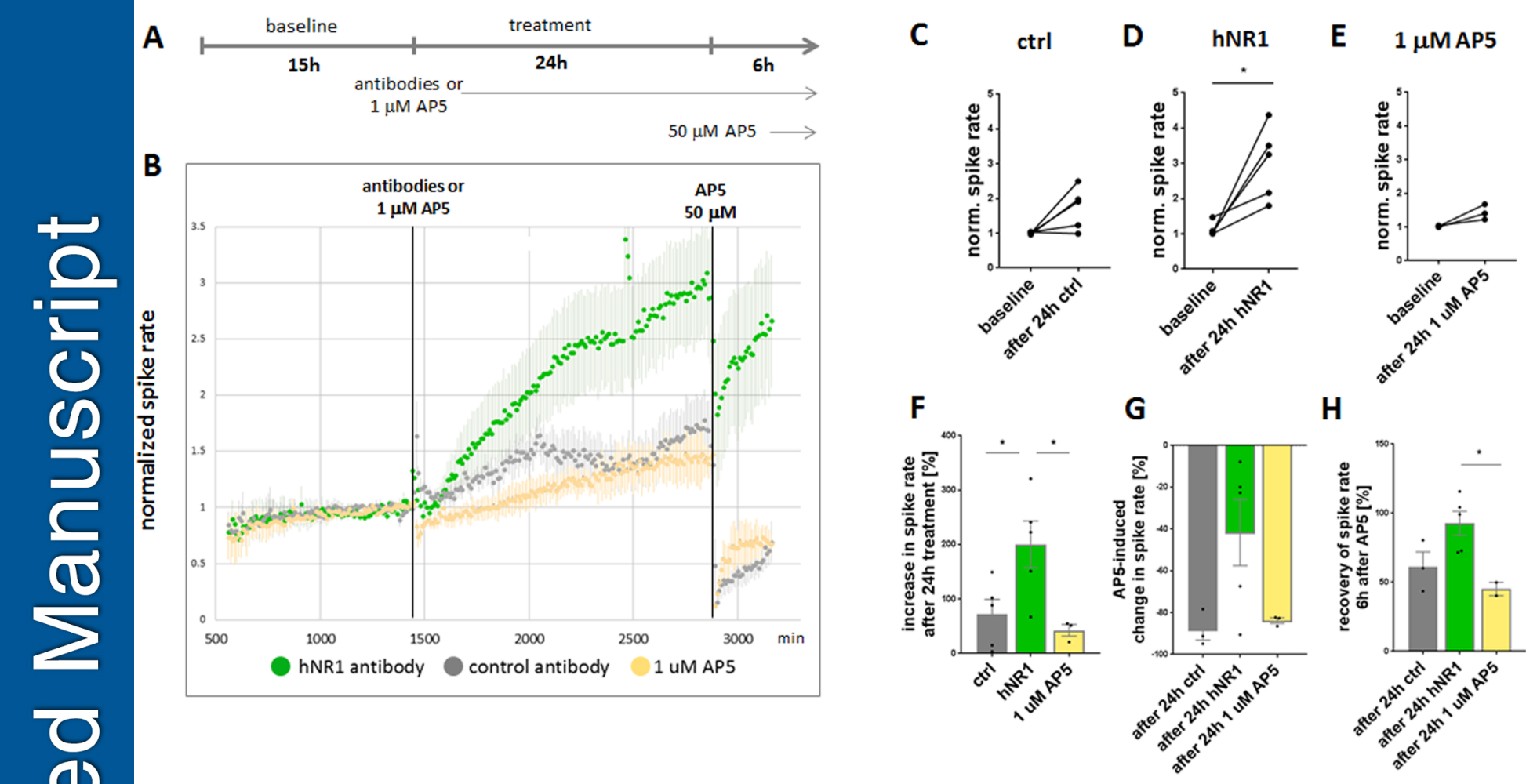

H
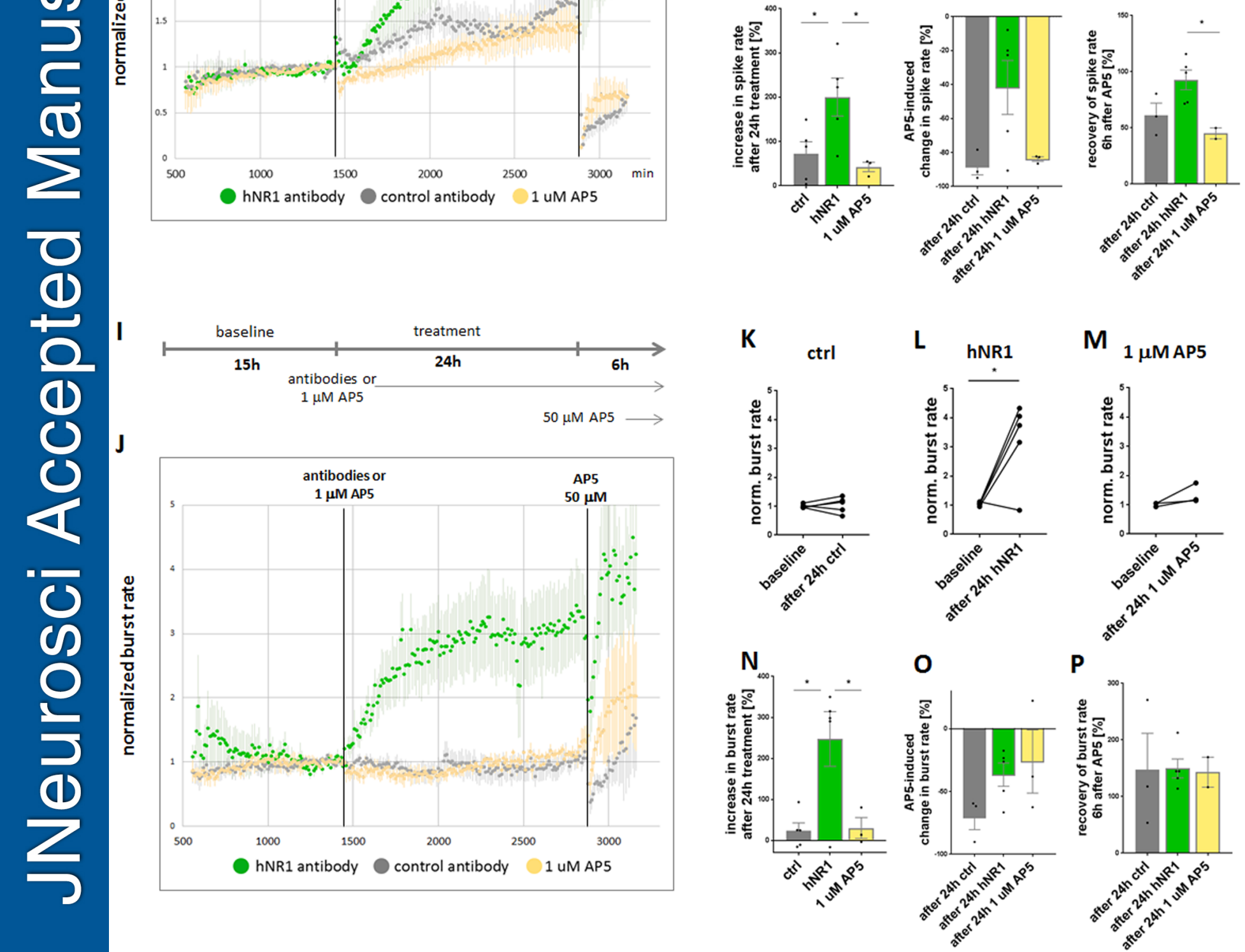

M $1 \mu$ M AP5
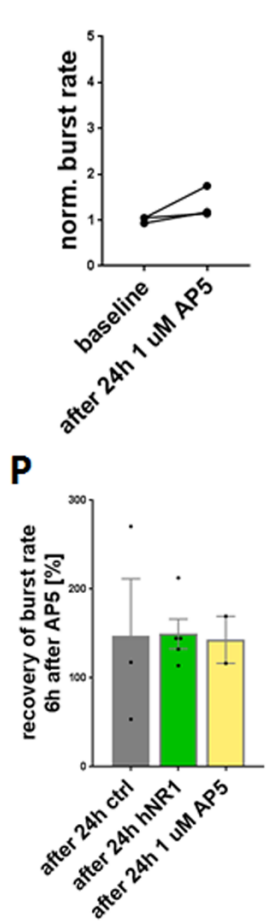

Figure 1 


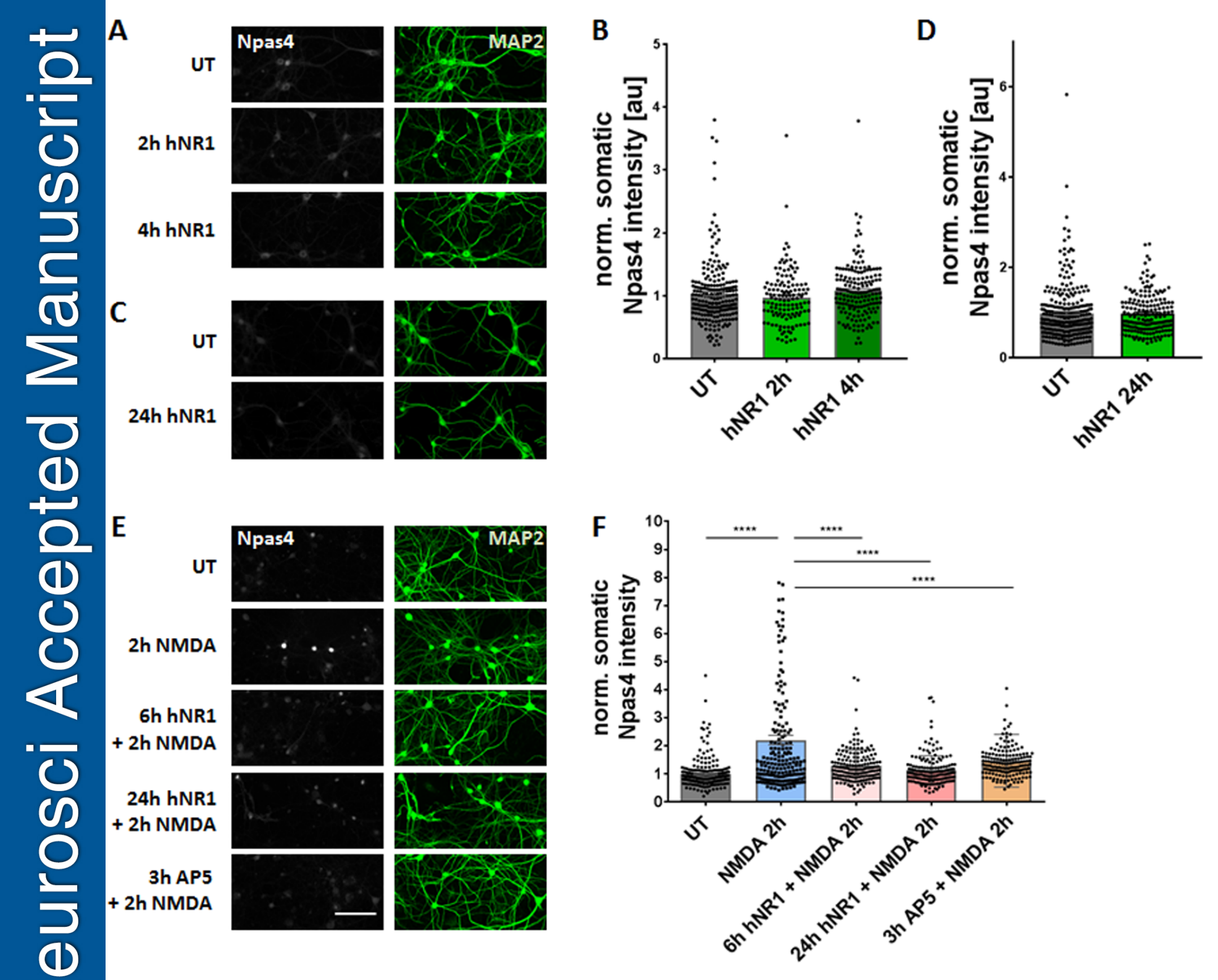

Figure 2 

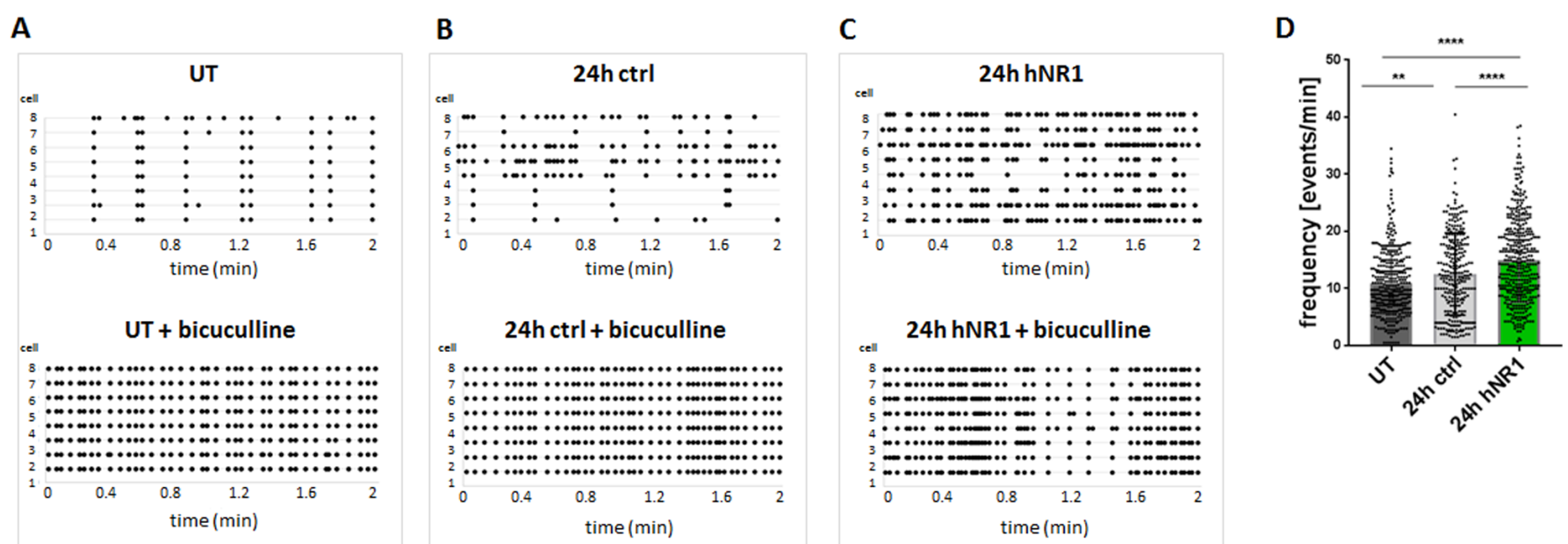

(d)

$\sqrt{10}$

(d)

E

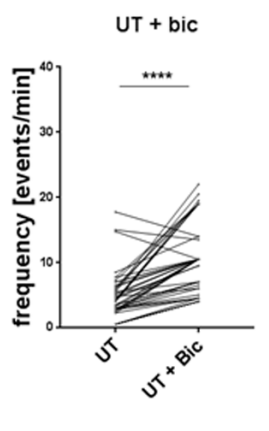

F

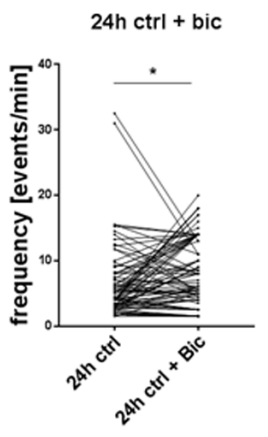

24h hNR1 + bicuculline

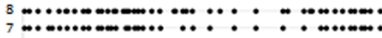

5 $4 . . . . .0-10$

$\begin{array}{lllll} & 0.4 & 0.8\end{array}$ time (min)

$\mathbf{G}$

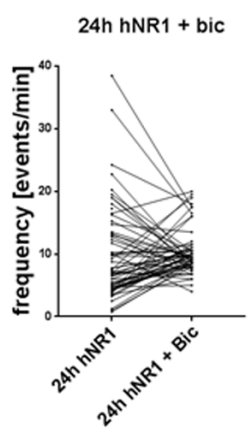

Figure 3 
mIPSCs

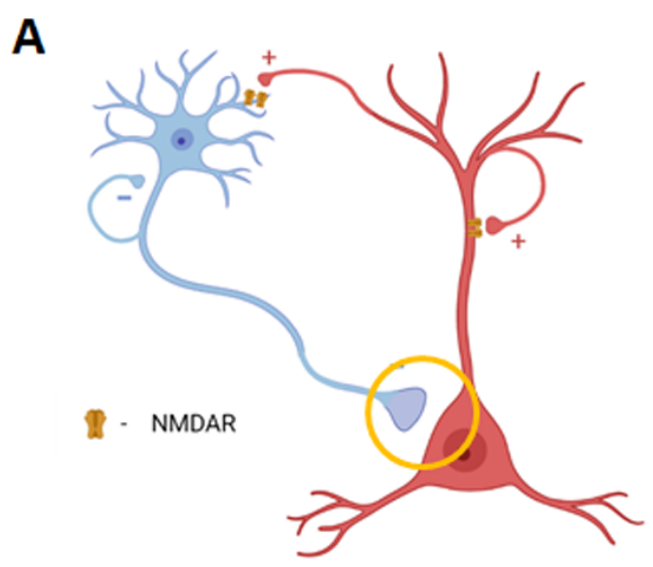

B

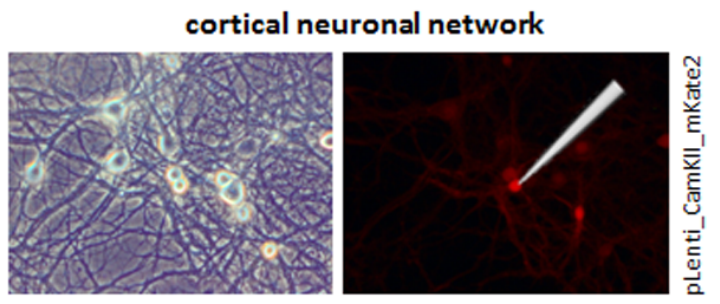

$\frac{0}{0}$

0
0
0

(a)

0

0

$<$

GABAR pool on excitatory neuron

C

ctrl

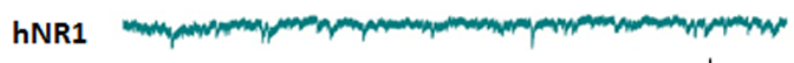

$\prod_{1 \mathrm{~s}} 100 \mathrm{pA}$

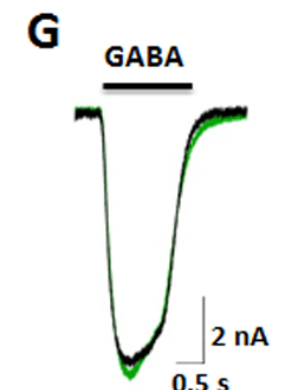

$\mathrm{H}$

mIPSCs
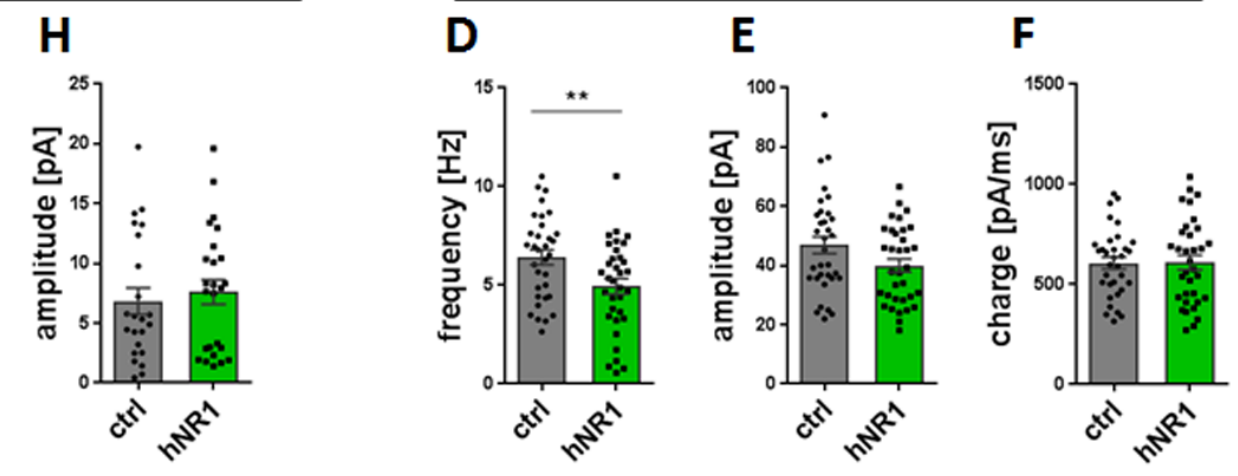

\begin{tabular}{l}
0 \\
0 \\
0 \\
$\frac{2}{3}$ \\
2 \\
\hline 3
\end{tabular}

ctrl

hNR1

inting

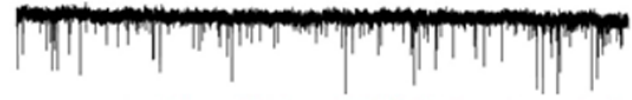

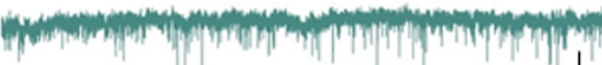

$\frac{1 \mathrm{~s}}{10 \mathrm{pA}}$
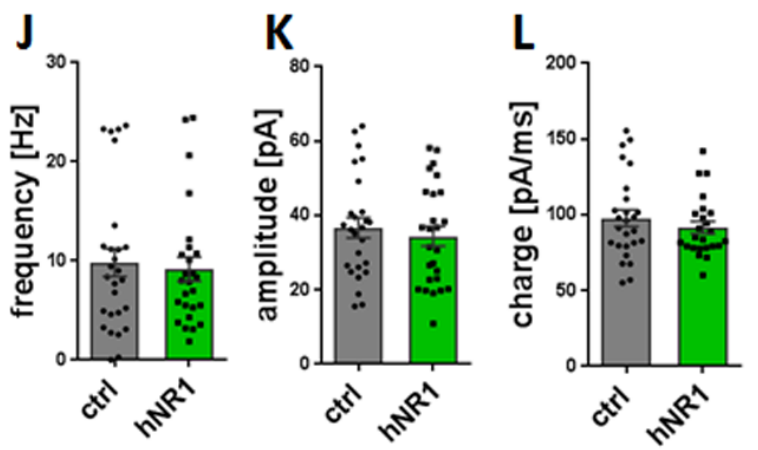

Figure 4

\section{mEPSCs}

M

onto excitatory neurons

ctrl

hNR1

$\underset{1 \mathrm{~s}}{ } 50 \mathrm{pA}$

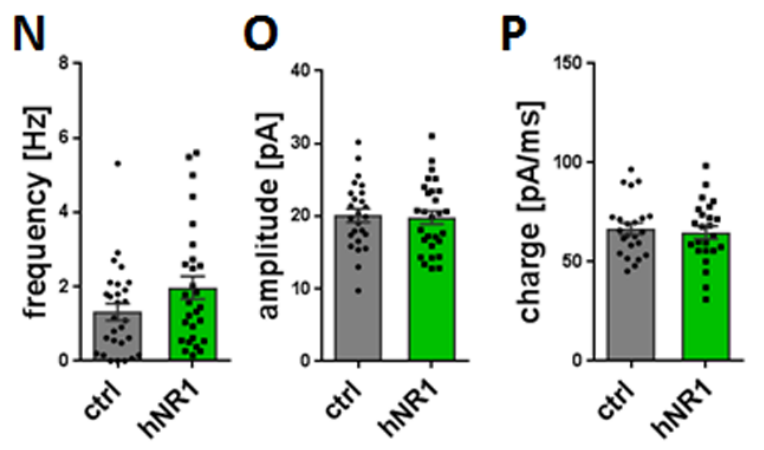


hippocampus - excitatory neurons

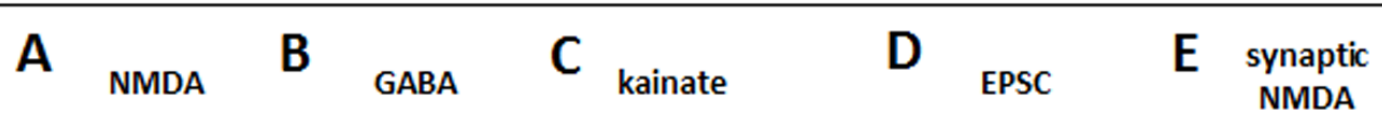
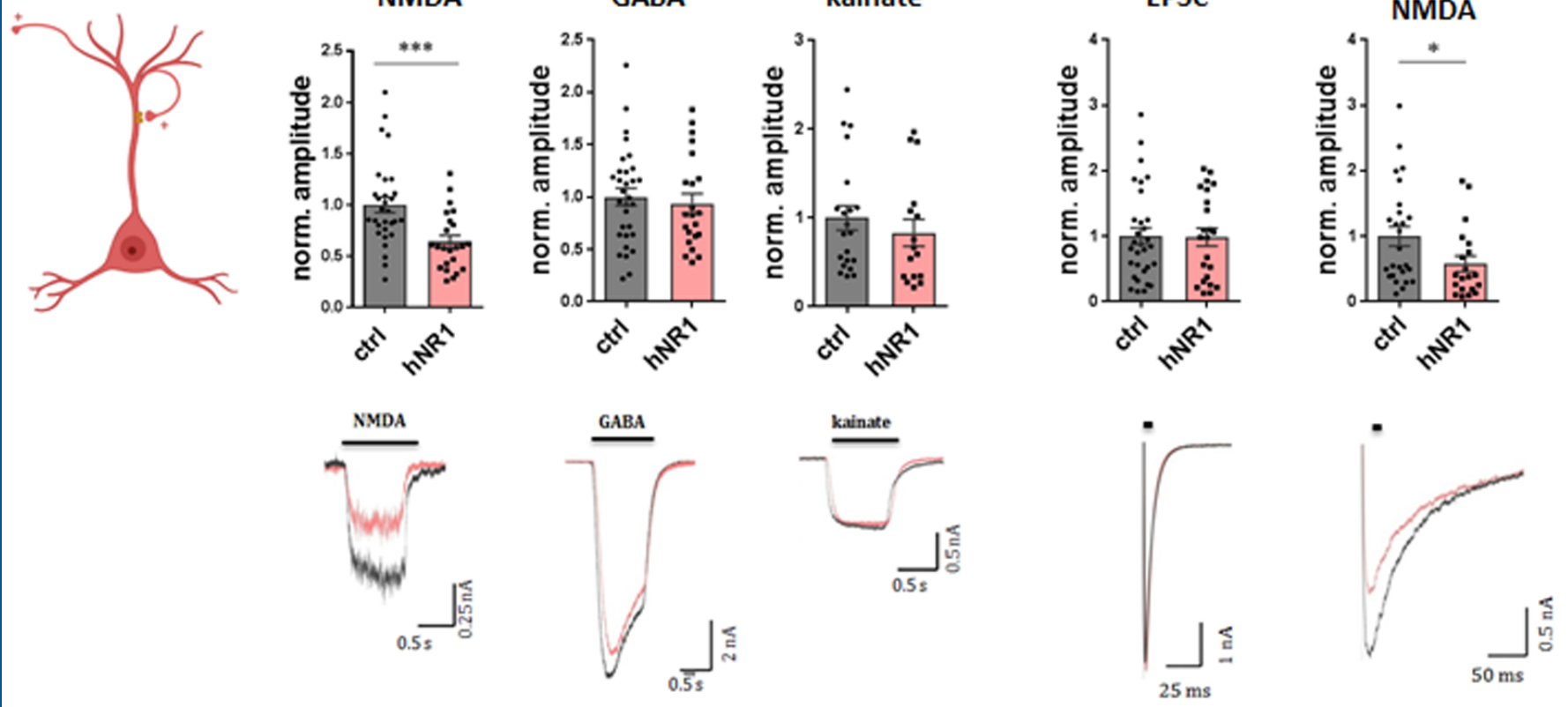

Figure 6 

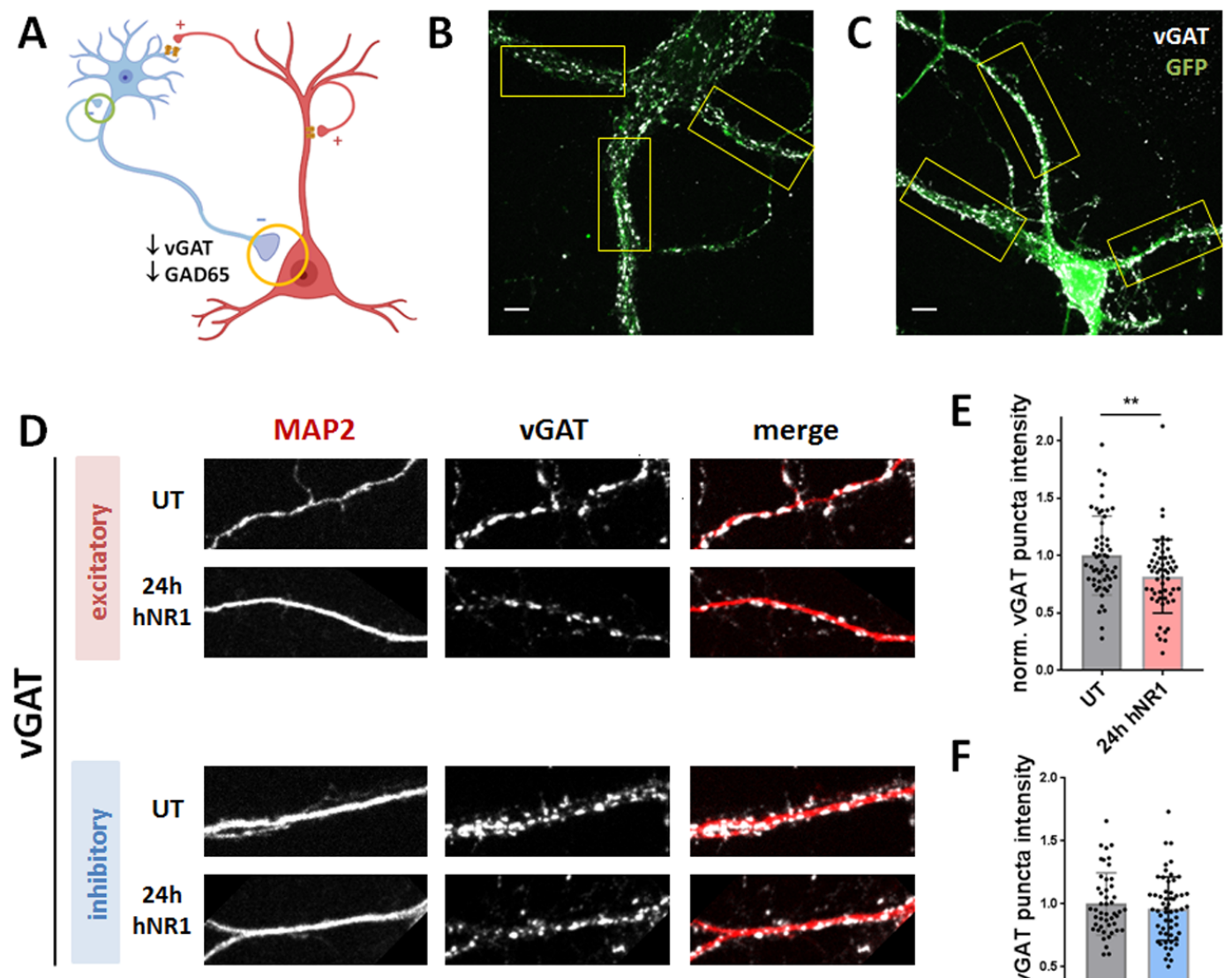

F
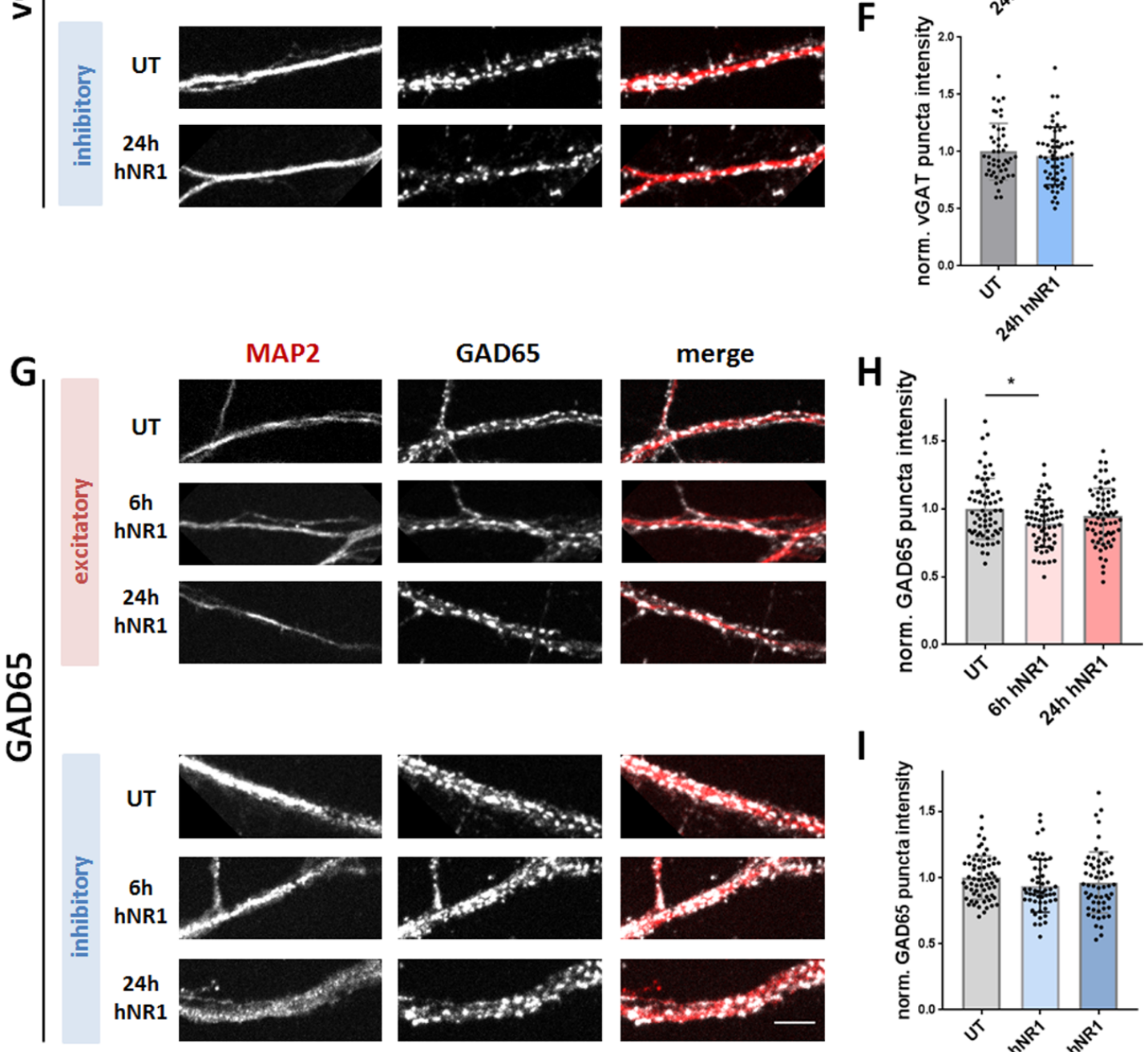

I

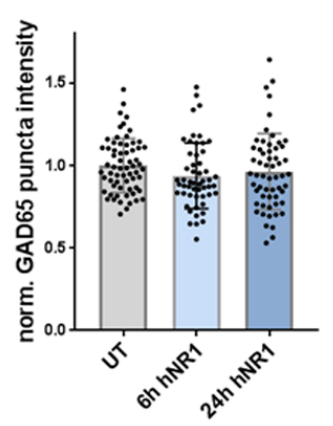

Figure 7 
A
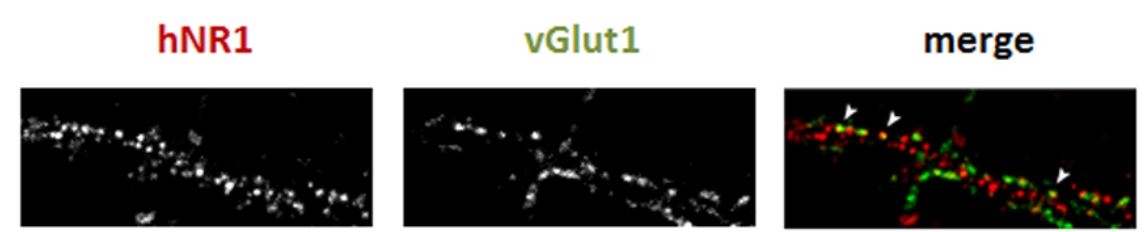

社
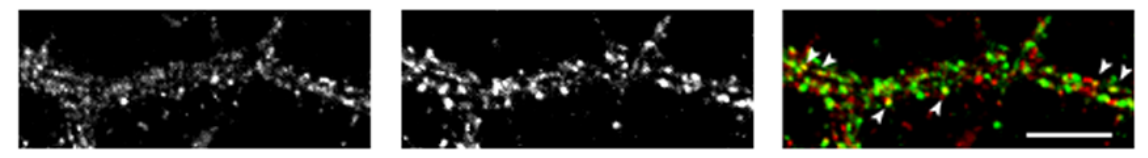

क

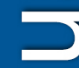

c

(1)

$\geq$

0

(1)

$+$

잉

(1)

0

0

$<$

c)

\&

3

(1)

D

C

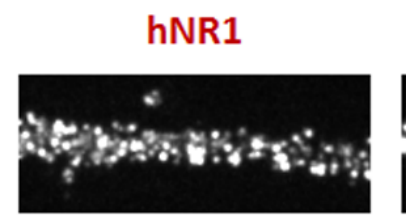

$\frac{ \pm}{\frac{2}{2}}$
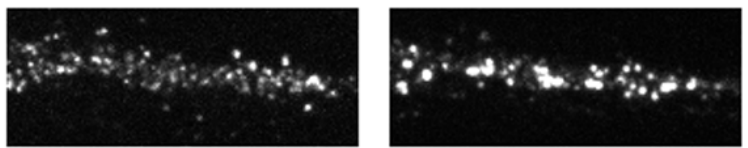

gephyrin

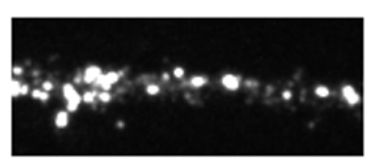

hNR1 gephyrin
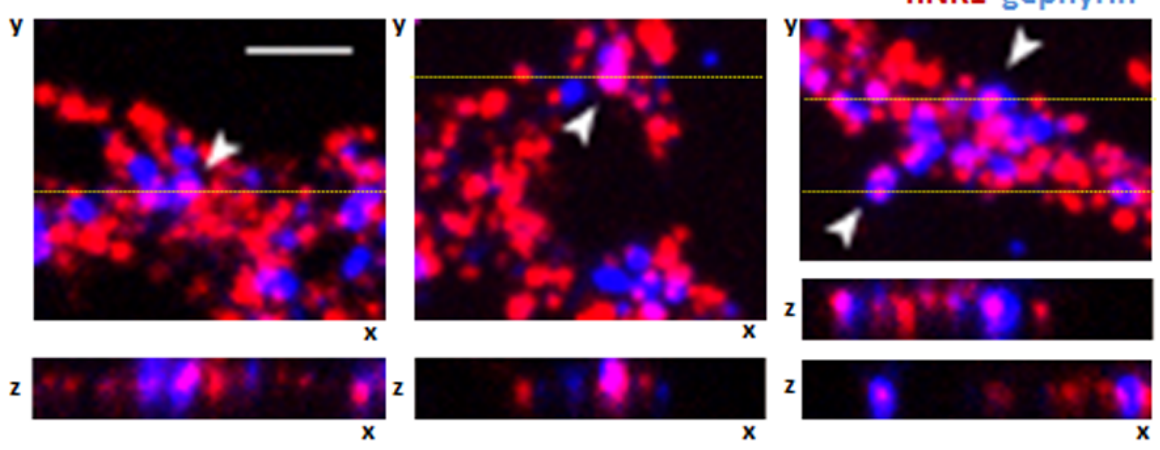

B

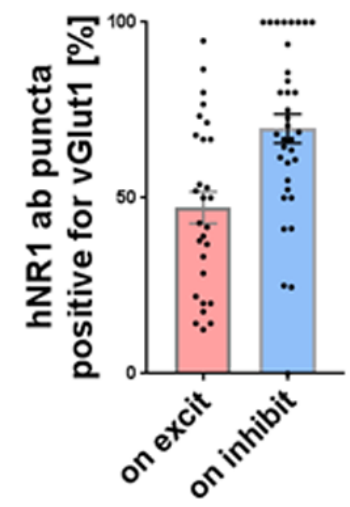

E

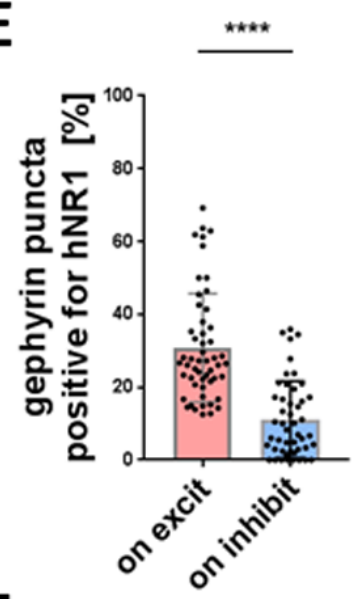

F

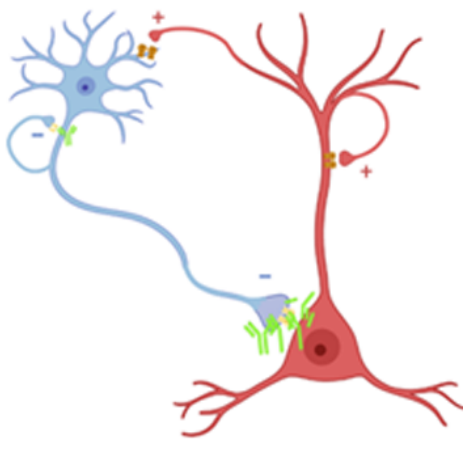

Figure 8 

(C) $\downarrow$ NMDA currents
(B) unchanged
mEPSCs
- pre-NMDAR

- post-NMDAR

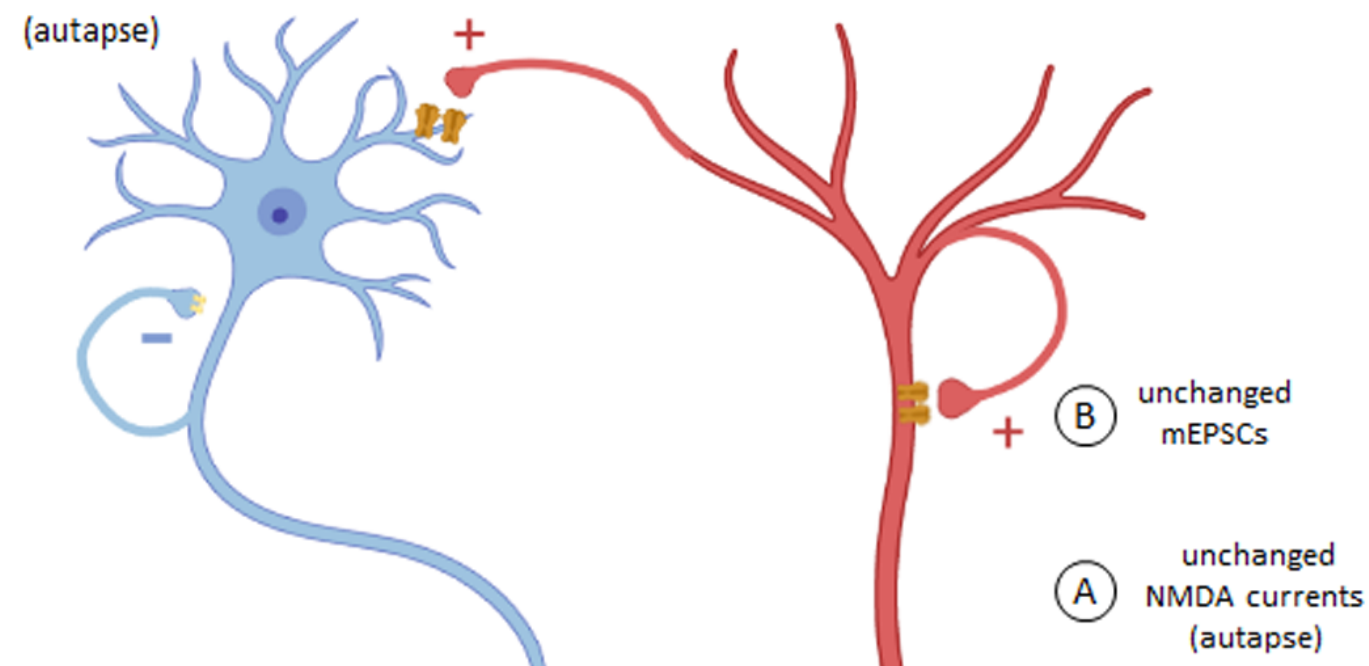

(F) $\downarrow$ vGAT

(G) $\downarrow$ GAD65

(D) $\downarrow$ freq mIPSCs

(E) $\downarrow$ amp IPSCs (autapse)

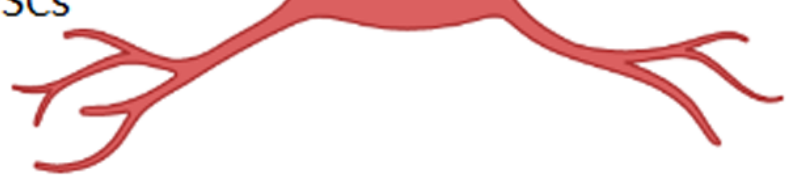

(1) $\uparrow$ network firing (I) $\uparrow$ network bursting

Figure 9 